\title{
ON THE LATTICE OF RECURSIVELY ENUMERABLE SETS
}

\author{
BY \\ A. H. LACHLAN
}

This paper presents some new theorems concerning recursively enumerable (r.e.) sets. The aim of the paper is to advance the search for a decision procedure for the elementary theory of r.e. sets. More precisely, an effective method is sought for deciding whether or not an arbitrary sentence formulated in the lower predicate calculus with sole relative symbol $\subseteq$ is true of the r.e. sets. The main achievement of the paper is the characterisation of the $h h$-simple sets as those coinfinite r.e. sets whose r.e. supersets form a Boolean algebra.

The reader is referred to Davis's book [1] for basic information about the partial recursive (p.r.) functions and about r.e. sets. Other background material required for a proper understanding of the present paper consists of [8], [3, Theorem 2], [10, Introduction and §4], and [5] where the contributions have been listed in their natural order. We take the formulation of the lower predicate calculus given in Abraham Robinson [9].

Natural numbers are denoted by lower case Roman letters and sets of them by lower case Greek letters. The empty set is denoted by $\varnothing$ and the set of all natural numbers by $\nu$. The complement of any set $\alpha$ is denoted by $\alpha^{\prime} ; \alpha$ is called cofinite or coinfinite just if $\alpha^{\prime}$ is finite or infinite respectively. For sets $\alpha, \beta$ we write $\alpha \simeq \beta$ just if the set $(\alpha-\beta) \cup(\beta-\alpha)$ is finite; otherwise we write $\alpha \npreceq \beta$. By function we mean a map of some subset of $\nu \times \nu \times \cdots \times \nu$ into $\nu$; functions will be denoted by upper case Roman letters as will relations on the natural numbers. The informal logical signs used are $\vee, \&, \rightarrow, \neg,(x),(E x), \Leftrightarrow$ which are to be read as "or", "and", "implies", "not", "for all $x$ ", "there exists $x$ ", "is equivalent to" respectively. Let $\mathscr{A}$ be a finite class of propositions; then $\& \mathscr{A}, \vee \mathscr{A}$ denote their conjunction and disjunction respectively. We use $\{A(x) \mid B(x)\}$ to denote the class of objects $A(a)$ for which $B(a)$ holds. If $\alpha$ is a finite set of natural numbers then $\sup \alpha$ denotes the greatest member of $\alpha$; otherwise sup $\alpha$ is to be $\infty$.

The plan of the paper is as follows. In the first section we give a brief discussion of the elementary theory of r.e. sets and prove that its decision problem is of the same degree as that of the elementary theory of the lattice obtained by taking the equivalence classes of r.e. sets with respect to $\simeq$. In $\$ 2$ we prove the main theorem which states: if $\alpha$ is an r.e. subset of an r.e. set $\beta$ then either there exists a recursive subset $\delta$ of $\beta$ such that $\alpha \cup \delta=\beta$ or there exists a recursive sequence $\left\{\delta_{i}\right\}$ of disjoint finite subsets of $\beta$ such that $\delta_{i}-\alpha$ is nonempty for all $i$. This theorem was inspired by

Received by the editors November 3, 1965 and, in revised form, November 16, 1966. 
Myhill's review [7] of Yates [10]. Yates pointed out [10, p. 344] that if $\mu_{1} \ldots, \mu_{n}$ are maximal sets such that any pair differ by an infinite set then any class of disjoint r.e. sets, each member of which has infinite intersection with $\left(\mu_{1} \cap \cdots \cap \mu_{n}\right)^{\prime}$, has cardinality $\leqq n$. Myhill in his review introduced the notion of covering number where the covering number of a set $\alpha$ is

sup \{cardinality of $\mathfrak{A} \mid \mathfrak{A}$ is a class of disjoint r.e. sets each having infinite intersection with $\alpha\}$.

Myhill claimed for Yates firstly that an r.e. set $\alpha$ is maximal if and only if the covering number of $\alpha^{\prime}$ is 1 , and secondly that an r.e. set $\alpha$ is $h h$-simple if and only if the the covering number of $\alpha^{\prime}$ is finite. Neither of these claims was justified. The result of Yates mentioned above may be restated as follows: if $\mu_{1}, \ldots, \mu_{n}$ are maximal r.e. sets such that any two differ by an infinite set, then $\left(\mu_{1} \cap \cdots \cap \mu_{n}\right)^{\prime}$ has covering number $n$. From the main theorem we shall deduce the converse of this which for $n=1$ justifies Myhill's first claim. Another consequence of the main theorem is the following characterisation of $h h$-simple sets: an r.e. set $\alpha$ is hh-simple if and only if $\alpha \cup \beta^{\prime}$ is r.e. for every r.e. set $\beta$. It follows that the intersection of two $h$ h-simple sets is also $h h$-simple. Thus with any $h h$-simple set $\alpha$ we can associate the Boolean algebra formed by the lattice of r.e. supersets of $\alpha$; this lattice we denote by $\mathscr{L}(\alpha)$ and the lattice formed from it by taking equivalence classes with respect to $\simeq$ we denote by $\mathscr{L}^{*}(\alpha)$.

In $\S 3$ we prove the falsity of Myhill's second claim by constructing an $h h$-simple set $\eta$ which has no maximal superset. In fact $\eta$ has the property that, if $\beta$ is any r.e. superset of $\eta$ then there exist r.e. supersets $\beta_{1}, \beta_{2}$ of $\eta$ such that $\beta_{1} \cup \beta_{2}=\beta, \beta_{1} \cap \beta_{2}$ $=\eta$, and such that

$$
\beta_{1} \simeq \eta \vee \beta_{2} \simeq \eta \rightarrow \beta \simeq \eta
$$

This property characterises $\mathscr{L}(\eta)$.

In $\$ 4$ the method of constructing $\eta$ in $\$ 3$, itself derived from Friedberg's construction of a maximal set [3, p. 312], is adapted to provide the following characterisation of the Boolean -algebras associated with $h h$-simple sets. Let $R$ be a binary $\exists \forall \exists$-relation, that is, let there be a recursive relation $S$ such that

$$
R(x, y) \Leftrightarrow(E u)(v)(E w) S(x, y, u, v, w) .
$$

Suppose further that $R$ is reflexive and transitive. Consider the class of sets $\mathscr{A}=\left\{\alpha_{0}, \alpha_{1}, \ldots\right\}$ where

$$
\alpha_{i}=\{x \mid R(x, i) \& R(i, x)\}
$$

and define $\leqq$ on $\mathscr{A}$ by

$$
\alpha_{i} \leqq \alpha_{j} \Leftrightarrow R(i, j)
$$


The class $\mathscr{A}$ is partially ordered by $\leqq$ because $R$ is reflexive and transitive. For some choices of $R$ the class $\mathscr{A}$ partially ordered by $\leqq$ forms a Boolean algebra, that is, $\mathscr{A}$ is a distributive lattice having a least element and a greatest element and such that every element has a complement. Any such Boolean algebra is called a $\exists \forall \exists-$ Boolean algebra provided that there are recursive functions $U, I$ such that for all $x, y$

$$
\alpha_{U(x, y)}=\alpha_{x} \cup \alpha_{y}, \quad \alpha_{I(x, y)}=\alpha_{x} \cap \alpha_{y} .
$$

We shall show that if $\alpha$ is hh-simple then $\mathscr{L}^{*}(\alpha)$ is isomorphic to a $\exists \forall \exists$-Boolean algebra, and conversely that if $\mathscr{A}$ is a $\exists \forall \exists-B o o l e a n$ algebra then there exists an hh-simple set $\alpha$ such that $\mathscr{L}^{*}(\alpha)$ is isomorphic to $\mathscr{A}$.

In $\$ 5$ are discussed some problems concerned with simple but non- $h h$-simple r.e. sets. It is proved that if $\alpha$ is any nonrecursive r.e. set then there exists an r.e. subset $\beta$ of $\alpha$ such that $\alpha-\beta$ is infinite and such that for any r.e. complement $\omega$ of $\alpha$ we have $\beta \cup \omega \simeq \nu$. From this and the main theorem is obtained an alternative to the construction by Martin [5] of an r.e. set which is coinfinite and which has no $h h$-simple superset. We shall also construct an $r$-maximal set with no maximal superset. An $r$-maximal set is a coinfinite r.e. set $\alpha$ such that for every recursive set $\rho$ either $\rho \cap \alpha^{\prime}$ or $\rho^{\prime} \cap \alpha^{\prime}$ is finite. This counterexamples the conjecture of R. W. Robinson [10] that every $r$-maximal set has a maximal superset.

Finally, in $\S 6$ we mention some problems concerning the elementary properties of r.e. sets which seem both interesting and within the reach of present methods.

1. The elementary theory of r.e. sets. We shall use the terminology and formal language $L$ of Abraham Robinson [9]. However, we shall only use $L$ when we have in mind a particular lattice $\mathscr{L}$. Which lattice $\mathscr{L}$ is will be clear from the context. The object symbols of $L$ are to be in fixed (1-1) correspondence $C$ with the elements of $\mathscr{L}$, the only relative symbol is to be the 2 -place relative symbol $Q($,$) . The$ dummy symbols of $L$ are to be $x_{1}, x_{2}, \ldots$; we shall also use these symbols as informal variables. Moreover, we shall be concerned with only one semantic interpretation of $L$, namely the one in which the structure is $\mathscr{L}$, in which the object symbols are mapped by $C$, and in which $Q($,$) is mapped onto the inclusion$ relation of $\mathscr{L}$. Below, sentence means sentence containing no object symbols, and predicate means predicate containing no object symbols.

Let $X$ be any predicate and $a_{1}, \ldots, a_{n}$ be any object symbols, then $X\left(a_{1}, \ldots, a_{n}\right)$ denotes the well-formed formula obtained by substituting $a_{1}, \ldots, a_{n}$ for $x_{1}, \ldots, x_{n}$ respectively in $X$. Let $R$ be a relation of order $n$ on $\mathscr{L}$. The predicate $X$ is said to define $R$ just if none of the variables $x_{n+1}, x_{n+2}, \ldots$ occurs free in $X$ and if for any object symbols $a_{1}, \ldots, a_{n}, X\left(a_{1}, \ldots, a_{n}\right)$ holds in $\mathscr{L}$ if and only if $R\left(a_{1}^{*}, \ldots, a_{n}^{*}\right)$ where $a_{1}, \ldots, a_{n}$ correspond to the respective elements $a_{1}^{*}, \ldots, a_{n}^{*}$ of $\mathscr{L}$. A relation on $\mathscr{L}$ is called elementary just if there is a predicate which defines it.

We suppose given a particular Gödel numbering of the well-formed formulas of $L$. 
Denote the lattice of r.e. sets of $\mathscr{R}$, we define certain relations on $\mathscr{R}$ as follows:

$$
\begin{gathered}
\operatorname{In}(x, y, z) \Leftrightarrow x \cap y=z, \\
\operatorname{Un}(x, y, z) \Leftrightarrow x \cup y=z, \\
\operatorname{Emp}(x) \Leftrightarrow x=\varnothing, \quad \operatorname{All}(x) \Leftrightarrow x=v, \\
\operatorname{Rec}(x) \Leftrightarrow x \text { is recursive, } \\
\operatorname{Fin}(x) \Leftrightarrow x \text { is finite, } \\
\operatorname{Sim}(x) \Leftrightarrow x \text { is simple, } \\
\operatorname{Max}(x) \Leftrightarrow x \text { is maximal. }
\end{gathered}
$$

Each of these relations is elementary. For the first four the following are corresponding predicates:

$$
\begin{gathered}
Q\left(x_{3}, x_{1}\right) \wedge Q\left(x_{3}, x_{2}\right) \wedge\left(\forall x_{4}\right)\left[Q\left(x_{4}, x_{1}\right) \wedge Q\left(x_{4}, x_{2}\right) . \supset Q\left(x_{4}, x_{3}\right)\right], \\
Q\left(x_{1}, x_{3}\right) \wedge Q\left(x_{2}, x_{3}\right) \wedge\left(\forall x_{4}\right)\left[Q\left(x_{1}, x_{4}\right) \wedge Q\left(x_{2}, x_{4}\right) . \supset Q\left(x_{3}, x_{4}\right)\right], \\
\left(\forall x_{2}\right)\left[Q\left(x_{1}, x_{2}\right)\right], \quad\left(\forall x_{2}\right)\left[Q\left(x_{2}, x_{1}\right)\right] .
\end{gathered}
$$

We can construct in turn predicates corresponding to the last four relations by using the equivalences:

$$
\begin{aligned}
\operatorname{Rec}(x) & \Leftrightarrow(E y)(E z)(E w)[\operatorname{In}(x, y, z) \& \operatorname{Un}(x, y, w) \& \operatorname{Emp}(z) \& \operatorname{All}(w)], \\
\operatorname{Fin}(x) & \Leftrightarrow(y)[y \subseteq x \rightarrow \operatorname{Rec}(y)], \\
\operatorname{Sim}(x) & \Leftrightarrow(y)(z)[\operatorname{Emp}(z) \& \operatorname{In}(x, y, z) . \rightarrow \operatorname{Fin}(y)], \\
\operatorname{Max}(x) & \Leftrightarrow(y)(E z)(E w)[x \subseteq y \rightarrow: \operatorname{Fin}(z) \& \operatorname{All}(w) \&[\operatorname{Un}(x, z, y) \vee \operatorname{Un}(z, y, w)]] .
\end{aligned}
$$

By the decision problem for $\mathscr{L}$ we mean the problem of deciding whether an arbitrary sentence of $L$ holds in $\mathscr{L}$ or not. For precision we define the decision problem for $\mathscr{L}$ to be the degree of unsolvability $d(\mathscr{L})$ of the set

$$
D(\mathscr{L})=\{x \mid x \text { is the g.n. of a sentence which holds in } \mathscr{L}\} .
$$

We say the decision problem for $\mathscr{L}$ is solvable just if $\boldsymbol{d}(\mathscr{L})=\boldsymbol{O}$, where $\boldsymbol{O}$ is the degree of the recursive sets. We say the decision problem for $\mathscr{L}$ is reducible to that for $\mathscr{M}$ if $d(\mathscr{L}) \leqq d(\mathscr{M})$.

It is apparent from the list of elementary relations on $\mathscr{R}$ given above that the decision problem for $\mathscr{R}$ cannot be easily proved solvable. For even the existence of a maximal set is by no means trivial. Also, if $\mathscr{R}$ has unsolvable decision problem, to demonstrate this we shall have to gather much more information about the elementary properties of r.e. sets than is presently available.

Let $\mathscr{N}$ denote the lattice formed by all subsets of the natural numbers, and let. $\mathscr{L}$ be any sublattice of $\mathscr{N}$. Suppose further that $\mathscr{L}$ is closed under addition and subtraction of finite sets, i.e., if $\lambda$ is in $\mathscr{L}$ and $\phi$ is finite then both $\lambda \cup \phi$ and $\lambda-\phi$ are in $\mathscr{L}$ also. We denote by $\mathscr{L}^{*}$ the lattice whose elements are the equivalence classes of members of $\mathscr{L}$ under $\simeq$. We shall now prove that the decision problem for $\mathscr{L}$ can be reduced to that for $\mathscr{L}^{*}$. 
Theorem 1. If $\mathscr{L} \subseteq \mathscr{N}$ and $\mathscr{L}^{*} \subseteq \mathscr{N}^{*}$ then the decision problem for $\mathscr{L}$ is reducible to that for $\mathscr{L}^{*}$.

Proof. Suppose that the hypothesis of the theorem is satisfied, then $\mathscr{L}$ is closed under the addition and subtraction of finite sets because $\mathscr{L}^{*} \subseteq \mathscr{N}^{*}$. If $\mathscr{L}$ has a greatest element it contains $\nu$, if it has a least element it contains $\varnothing$. We shall suppose below that $\mathscr{L}$ contains both $\nu$ and $\varnothing$; we shall discuss at the end of the proof what modifications are necessary in case one or both of $\nu, \varnothing$ are absent from $\mathscr{L}$.

We let $y$ denote the $k$-tuple $y_{1}, \ldots, y_{k}$ of variables which range through $\mathscr{L}$. Let $\kappa=\{1,2, \ldots, k\}$ and for each subset $\iota$ of $\kappa$ let $\boldsymbol{y}(\iota)$ denote

$$
\bigcap\left\{y_{i} \mid i \in \iota\right\} \cap \bigcap\left\{y_{i}^{\prime} \mid i \in \kappa-\imath\right\} .
$$

For each natural number $m$ let $C_{m}, C_{\geqq m}$ denote the respective relations on $\mathscr{L}$ defined by

$$
\begin{gathered}
C_{m}(y) \Leftrightarrow y \text { has cardinality } m, \\
C_{\geqq m}(y) \Leftrightarrow y \text { has cardinality } \geqq m .
\end{gathered}
$$

By an $A$-relation of order $k$ we mean a relation $R$ of order $k$ on $\mathscr{L}$ such that $R(y)$ is equivalent to

$$
\&\left\{C_{\iota}(y(\iota)) \mid \iota \subseteq \kappa\right\}
$$

where each $C_{\iota}$ has one of the forms $C_{m}, C_{\geqq m}$ or is identically false. By a B-relation of order $k$ we mean a relation $R$ of order $k$ on $\mathscr{L}$ such that $R(y)$ is equivalent to $S(\{\boldsymbol{y}\})$ where $S$ is a relation of order $k$ on $\mathscr{L}^{*}$ and where $\{\boldsymbol{y}\}$ denotes $\left\{y_{1}\right\}, \ldots,\left\{y_{k}\right\}$, these being the respective members of $\mathscr{L}^{*}$ to which $y_{1}, \ldots, y_{k}$ in $\mathscr{L}$ belong. We define the $B$-relation corresponding to the $A$-relation (1) to be

$$
\&\left\{F_{\iota}(\{\boldsymbol{y}\}) \mid \iota \subseteq \kappa\right\},
$$

where $F_{\iota}$ is identically false if $C_{\iota}$ is, $F_{\iota}$ is identically true if $C_{\iota}$ has the form $C_{\geqq m}$ and where $F_{c}(z)$ is

$$
\bigcap\left\{z_{i} \mid i \in \iota\right\} \subseteq \bigcup\left\{z_{i} \mid i \in \kappa-\imath\right\}
$$

otherwise. The left-hand side of this last inclusion is to be $\{\nu\}$ when $\iota=\varnothing$, and the right-hand side is to be $\{\varnothing\}$ when $\iota=\kappa$. Notice that (3) holds for $z=\{y\}$ if and only if $y(\iota)$ is finite. Notice also that (1) implies (2).

By an $A B$-relation of order $k$ we mean a relation $R$ of order $k$ on $\mathscr{L}$ such that $R(y)$ is equivalent to

$$
\bigvee\left\{A_{i}(y) \& B_{i}(y) \mid i=0,1, \ldots, p\right\}
$$

where each $A_{i}$ is an $A$-relation and each $B_{i}$ is a $B$-relation. Notice that any $A$-relation of order $k$ is an $A B$-relation of order $k$, and similarly for any $B$-relation of order $k$.

We give Gödel numbers to the $A$-relations of order $k$ so that from the g.n. of $R$ we can effectively find all the relations $C_{\iota}$ in (1), and vice-versa. A number $e$ is 
said to be the g.n. of a $B$-relation of order $k$ just if the well-formed formula of $L$ with g.n. $e$ defines a corresponding relation of order $k$ on $\mathscr{L}^{*}$. We give g.n.'s to the $A B$-relations of order $k$ so that from the g.n. of the $A B$-relation expressed by (4) we can effectively find $p$ and g.n.'s of $A_{0}, A_{1}, \ldots, A_{p}, B_{0}, B_{1}, \ldots, B_{p}$ respectively, and vice-versa. Now when we say "given an $A-, B-$, or $A B$-relation" we mean given its g.n., and when we say we can effectively find an $A-, B-$, or $A B$-relation we mean we can effectively find its g.n.

We now develop certain closure properties of the class of $A B$-relations. It is clear that given two $A B$-relations we can effectively find their disjunction as an $A B$-relation. We shall prove that the conjunction of $A B$-relations can also be effectively found as an $A B$-relation. Suppose then that for $j=0,1$ we have $R^{j}(y)$ equivalent to

$$
\bigvee\left\{A_{i}^{j}(y) \& B_{i}^{j}(y) \mid i=0,1, \ldots, p^{j}\right\}
$$

where the $A_{i}^{j}$ are $A$-relations and the $B_{i}^{j}$ are $B$-relations. Now $R^{0}(y) \& R^{1}(y)$ is equivalent to the disjunction of all the expressions of the form

$$
A_{g}^{0}(y) \& B_{g}^{0}(y) \& A_{h}^{1}(y) \& B_{h}^{1}(y) ; \quad 0 \leqq g \leqq p^{0}, \quad 0 \leqq h \leqq p^{1} .
$$

Thus it is clearly sufficient to show that given two $A$-relations we can effectively find their conjunction as an $A$-relation. Suppose then that for $j=0,1$ we have

$$
A^{j}(y) \Leftrightarrow \&\left\{C_{\imath}^{j}(y(\iota)) \mid \iota \subseteq \kappa\right\}
$$

where $C_{\iota}^{0}, C_{\iota}{ }^{1}$ can take the same forms as $C_{\iota}$ in (1). It is easy to see that $A^{0}(y) \&$ $A^{1}(y)$ is equivalent to (1) provided that the relations $C_{\iota}$ are chosen as follows. If $C_{\iota}^{0}, C_{\imath}^{1}$ are $C_{m}, C_{n}$ respectively, we take $C_{\iota}$ to be $C_{m}$ if $m=n$ and to be identically false otherwise. If $C_{\imath}^{0}, C_{\imath}^{1}$ are $C_{m}, C_{\geqq n}$ in some order then we choose $C_{\imath}$ to be $C_{m}$ if $m \geqq n$ and to be identically false otherwise. If $C_{\iota}^{0}, C_{\iota}^{1}$ are $C_{\geqq m}, C_{\geqq n}$ respectively, we take $C_{\iota}$ to be $C_{\geqq \max (m, n)}$. Finally, if one of $C_{\iota}^{0}, C_{\iota}^{1}$ is identically false we take $C_{\iota}$ to be identically false. Thus given the $A$-relations $A^{0}, A^{1}$ we can effectively find their conjunction as an $A$-relation; from above it follows that the same is true for $A B$-relations.

The negation of (4) can be written

$$
\&\left\{\neg A_{i}(y) \cdot \vee . \neg B_{i}(y) \mid i=0,1, \ldots, p\right\} .
$$

To show that we can find the g.n. of an $A B$-relation $R$ such that $R(y)$ is equivalent to (5) it is sufficient to show that given an $A$-relation we can effectively find its negation as an $A B$-relation, and that given a $B$-relation we can effectively find its negation as a $B$-relation. The latter is immediate, for to obtain the negation of a $B$ relation we need only take the negation of the corresponding elementary relation on $\mathscr{L}^{*}$. Now consider the $A$-relation expressed by (1); the negation of (1) is a disjunction of terms of the forms $\neg C_{m}(y(\alpha)), \neg C_{\geqq m}(y(\alpha))$ where $m$ is a natural number and $\alpha \subseteq \kappa$. We are neglecting the case in which one of the $C_{\imath}$ is identically 
false, because the identically true relation is obtained by taking each $C_{\iota}$ to be $C_{\geqq 0}$ in (1). Now for $m>0$

$$
\neg C_{m} \Leftrightarrow\left[C_{0} \vee C_{1} \vee \cdots \vee C_{m-1} \vee C_{\geqq m+1}\right]
$$

and

$$
\neg C_{\geqq m} \Leftrightarrow\left[C_{0} \vee C_{1} \vee \cdots \vee C_{m-1}\right] .
$$

Also, $\neg C_{0}$ is equivalent to $C_{\geqq 1}$, and $\neg C_{\geqq 0}$ is identically false. Thus it suffices to show that given $m$ and a subset $\alpha$ of $\kappa$ we can find an $A$-relation $R$ such that $R(y)$ is equivalent to whichever of $C_{m}(\boldsymbol{y}(\alpha)), C_{\geq m}(\boldsymbol{y}(\alpha))$ we choose. Now the former is equivalent to (1) when we take $C_{\iota}$ to be $C_{\geq 0}$ for $\iota \neq \alpha$ and $C_{\alpha}$ to be $C_{m}$, the latter is equivalent to (1) when we take $C_{\imath}$ to be $C_{\geq 0}$ for $\iota \neq \alpha$ and $C_{\alpha}$ to be $C_{\geq m}$. Hence the negation of an $A B$-relation is an $A B$-relation which can be found effectively.

Consider now an $A B$-relation $R$ of order $k+1$. We can suppose that $R(y, y)$ is

$$
\bigvee\left\{A_{i}(y, y) \& B_{i}(y, y) \mid i=0,1, \ldots, p\right\}
$$

where $A_{i}$ is an $A$-predicate and $B_{i}$ is a $B$-predicate for $0 \leqq i \leqq p$. We shall show that $(E y) R(y, y)$ can be effectively expressed in the form (4). Because an existential quantifier can be taken inside a disjunction it is sufficient to consider the case $p=0$. Thus we suppose that $R(y, y)$ is $A(y, y) \& B(y, y)$ where $A$ is an $A$-relation and $B$ is a $B$-relation; we suppose further that $B(y, y)$ implies the $B$-relation corresponding to $A$. There is no loss of generality, because $A$ implies the $B$-relation corresponding to it. Now $A(y, y)$ has the form

$$
\&\left\{C_{\iota}^{0}(y(\iota) \cap y), C_{\iota}^{1}\left(y(\iota) \cap y^{\prime}\right) \mid \iota \subseteq \kappa\right\}
$$

where $C_{\iota}^{0}, C_{\iota}^{1}$ have the same possible forms as $C_{\iota}$ in (1). We define an $A$-relation $A^{0}$ of order $k$ by the following specification of $C_{\imath}$ in (1). If $C_{\imath}^{0}, C_{\imath}^{1}$ are $C_{m}, C_{n}$ respectively we take $C_{\imath}$ to be $C_{m+n}$. If $C_{\imath}^{0}, C_{\imath}^{1}$ are $C_{\geq m}, C_{n}$ or $C_{\geqq m}, C_{\geqq n}$ in some order we take $C_{\iota}$ to be $C_{\geqq m+n}$. If one of $C_{\imath}^{0}, C_{\imath}^{1}$ is identically false we take $C_{\iota}$ to be identically false. We shall now prove that

$$
(E y) R(y, y) \Leftrightarrow\left[A^{0}(y) \&(E y) B(y, y)\right] .
$$

From left to right is easy; we have only to note that

$$
(\boldsymbol{y}(\iota) \cap y) \cup\left(\boldsymbol{y}(\iota) \cap y^{\prime}\right)=\boldsymbol{y}(\iota)
$$

so that the sum of the cardinalities of $y(\iota) \cap y$ and $y(\iota) \cap y^{\prime}$ is equal to the cardinality of $y(\imath)$. Suppose that the right-hand side holds for values $y_{1}=\omega_{1}, \ldots, y_{k}=\omega_{k}$ in $\mathscr{L}$, and let $\omega$ be a member of $\mathscr{L}$ such that $B(\omega, \omega)$. For each $\iota$ we shall define finite subsets $\phi_{\iota}, \psi_{\iota}$ of $\omega(\iota)$ such that, if $\omega_{\iota}=\left(\omega-\phi_{\iota}\right) \cup \psi_{\iota}$, then $C^{0}\left(\omega(\iota) \cap \omega_{\iota}\right)$ and $C^{1}\left(\omega(\iota) \cap \omega_{\imath}^{\prime}\right)$. If $C_{\iota}^{0}, C_{\iota}^{1}$ are $C_{m}, C_{n}$ then from $A^{0}(\omega)$ we have $C_{m+n}(\omega(\iota))$. We let $\psi_{\iota}$ consist of the $m$ least members of $\omega(\iota)$ and we let $\phi_{\iota}$ consist of the $n$ remaining members. If $C_{\iota}^{0}, C_{\iota}^{1}$ are $C_{\geqq m}, C_{n}$ respectively then from $A^{0}(\omega)$ we have $C_{\geqq m+n}(\omega(\iota))$. Also, since $B$ implies the $B$-relation corresponding to $A$ and since $C_{\iota}^{1}$ is $C_{n}$ we have 
from (3) that $\omega(\iota) \cap \omega^{\prime}$ is finite. Let $\phi_{\iota}$ consist of the $n$ least members of $\omega(\iota)$, let $\psi_{\iota}=\omega(\iota) \cap \omega^{\prime} \cap \phi_{\iota}^{\prime}$. If $C_{\iota}^{0}, C_{\iota}^{1}$ are $C_{m}, C_{\geqq n}$ respectively then by the same argument we have $C_{\geq m+n}(\omega(\iota))$ again and $\omega(\iota) \cap \omega$ finite. Let $\psi_{\iota}$ consist of the $m$ least members of $\omega(\iota)$, and let $\phi_{\iota}=\omega(\iota) \cap \omega$. If $C_{\iota}^{0}, C_{\iota}^{1}$ are $C_{\geqq m}, C_{\geqq n}$ respectively then from $A^{0}(\boldsymbol{\omega})$ we have $C_{\geqq m+n}(\boldsymbol{\omega}(\iota))$. Let $\phi_{\iota}$ consist of the $m+n$ least members of $\boldsymbol{\omega}(\iota)$, and let $\psi_{\iota}$ consist of the $m$ least members of $\omega(\iota)$. This completes the definition of $\phi_{\iota}$, $\psi_{\iota}$ for all $\iota \subseteq \kappa$. We define

$$
\omega^{0}=\left(\omega-\bigcup\left\{\phi_{\imath} \mid \iota \subseteq \kappa\right\}\right) \cup \bigcup\left\{\psi_{\imath} \mid \iota \subseteq \kappa\right\}
$$

Since all the sets $\omega(\iota)$ are disjoint we have $\omega(\iota) \cap \omega^{0}=\omega(\iota) \cap \omega_{\imath}$. Hence $C_{\iota}^{0}\left(\omega(\iota) \cap \omega^{0}\right)$ and $C_{\iota}^{1}\left(\omega(\iota) \cap\left(\omega^{0}\right)^{\prime}\right)$ for all $\iota \subseteq \kappa$, which means that $A\left(\omega, \omega^{0}\right)$. Also, since $\omega^{0}$ only differs finitely from $\omega$ we have $B\left(\omega, \omega^{0}\right)$. Thus the left side of (6) holds for $y=\omega$ which is what we had to prove. If the relation $B^{*}$ of order $k+1$ on $\mathscr{L}^{*}$ gives rise to $B$, then the elementary relation of order $k$ on $\mathscr{L}^{*}$ expressed by $(E z) B^{*}(z, z)$ gives rise to the relation of order $k$ on $\mathscr{L}$ expressed by $(E y) B(y, y)$. Therefore we can find an $A B$-relation $S$ of order $k$ such that $(E y) R(y, y)$ is equivalent to $S(y)$.

We have now shown that the class of $A B$-relations of order $k$ is effectively closed under $\vee, \&, \neg$; and that the application of an existential quantifier maps the class of $A B$-relations of order $k+1$ effectively into the class of $A B$-relations of order $k$.

Let $X$ be any sentence. We now show how to find a sentence $X^{*}$ such that $X^{*}$ holds in $\mathscr{L}^{*}$ if and only if $X$ holds in $\mathscr{L}$. From the theory of prenex normal forms $[9$, p. 8] we can suppose that $X$ has the form

$$
\left(\exists x_{2}\right) \sim\left(\exists x_{3}\right) \sim \cdots \sim\left(\exists x_{k}\right) Y,
$$

where $Y$ is a predicate containing no quantifiers and only the variables $x_{2}, x_{3}, \ldots$, $x_{k}$. Consider any subpredicate of $Y$; it is built up from parts $Q\left(x_{i}, x_{j}\right)$ by using $\wedge, \vee$, and $\sim$. Now $Q\left(x_{i}, x_{j}\right)$ defines the $A$-relation of order $k$ obtained by taking $C_{\iota}$ to be $C_{0}$ when $\iota$ contains $i$ but not $j$, and $C_{\iota}$ to be $C_{\geqq 0}$ otherwise. Further, if $Y_{1}, Y_{2}$ are both subpredicates of $Y$ defining $A B$-relations $R_{1}, R_{2}$ of order $k$ respectively, it is easy to see that $\left[Y_{1} \vee Y_{2}\right],\left[Y_{1} \wedge Y_{2}\right],\left[\sim Y_{1}\right]$ define the $A B$-relations $R_{1} \vee R_{2}, R_{1} \& R_{2}, \neg R_{1}$ respectively. Thus using the procedures worked out above we can effectively find from $X$ the $A B$-relation of order $k$ defined by $Y$; call this relation $R$. Further, we can effectively find an $A B$-relation $S$ of order one such that

$$
S(y) \Leftrightarrow\left(E y_{2}\right) \neg\left(E y_{3}\right) \neg \cdots \neg\left(E y_{k}\right) R\left(y, y_{2}, y_{3}, \ldots, y_{k}\right) .
$$

From the definition of $A B$-relation there are $A$-relations $A_{i}$ and elementary relations $B_{i}^{*}$ on $\mathscr{L}^{*}$ such that $S(y)$ is equivalent to

$$
\bigvee\left\{A_{i}(y) \& B_{i}^{*}(\{y\}) \mid i=1,2, \ldots, p\right\}
$$

We know that $S(y)$ is independent of $y$, because $Y$ does not contain $x_{1}$. Thus $X$ holds in $\mathscr{L}$ just if $S(\varnothing)$ holds. For each $i$ we can effectively decide $A_{i}(\varnothing)$. Also, for each $i$ we can effectively find a sentence which holds in $\mathscr{L}^{*}$ just if $B_{i}^{*}(\{\varnothing\})$ 
holds in $\mathscr{L}^{*}$. This is because the relation $z=\{\varnothing\}$ is elementary on $\mathscr{L}^{*}$. Thus given a g.n. of $X$ we can effectively find a g.n. of $X^{*}$ which holds in $\mathscr{L}^{*}$ just if $X$ holds in $\mathscr{L}$.

This completes the proof of the theorem except for discussion of the modifications which are necessary when one or both of $\varnothing, \nu$ are absent from $\mathscr{L}$. If $\varnothing$ is absent, we restrict $C_{\kappa}$ in (1) to be either $C_{\geqq 0}$ or identically false; if $\nu$ is absent, we restrict $C_{\varnothing}$ in (1) to be either $C_{\geqq 0}$ or identically false; if both $\varnothing, \nu$ are absent we make both restrictions. We make corresponding changes in the definition of $A^{0}$ in (6). In the last paragraph we also make the following adjustments if $\varnothing$ is absent from $\mathscr{L}$. If $\nu$ is present we just read $\nu$ for $\varnothing$. If both $\varnothing, v$ are absent, then because of the restrictions each $A_{i}$ in (7) is identically true or identically false. We can still find $X^{*}$ effectively because $X$ holds in $\mathscr{L}$ just if

$$
\bigvee\left\{(E z) B_{i}^{*}(z) \mid 1 \leqq i \leqq p \& A_{i} \text { identically true }\right\}
$$

holds in $\mathscr{L}^{*}$.

The above proof is just a straightforward elimination of quantifiers. Applying the same technique when we have the additional stipulation that $\mathscr{L}$ is a Boolean algebra we can prove that the only elementary relations on $\mathscr{L}$ are disjunctions of $A$-relations and consequently that $\mathscr{L}$ has solvable decision problem. This explains why there are no interesting elementary properties of the lattice of recursive sets.

From the theorem we see that in investigating the elementary theory of $\mathscr{R}$ we can confine attention to $\mathscr{R}^{*}$.

If $\mathscr{L} \subseteq \mathscr{N}$ and $\mathscr{L}^{*} \subseteq \mathscr{N}^{*}$ we can prove the converse of Theorem 1, i.e., that the decision problem for $\mathscr{L}^{*}$ is reducible to that for $\mathscr{L}$, provided that the relation $y_{1} \simeq y_{2}$ is an elementary relation on $\mathscr{L}$. Given a formula $X^{*}$ defining an elementary relation $R^{*}$ of order $k$ on $\mathscr{L}^{*}$ we can effectively find a formula $X$ defining $R$ on $\mathscr{L}$, where $R(y)$ is equivalent to $R^{*}\left(\left\{y_{1}\right\}, \ldots,\left\{y_{k}\right\}\right)$. The proof is by induction on the length of $X^{*}$. For the starting case consider $X^{*}$ of the form $Q\left(x_{i}, x_{j}\right)$ we take $X$ to be a formula defining the relation $R$ of order $k$ on $\mathscr{L}$ such that $R(y)$ is $y_{i} \cap y_{j}$ $\simeq y_{i}$. We can find such $X$, because the relation $\simeq$ is elementary on $\mathscr{L}$ by assumption. The induction step is immediate: corresponding to $X^{*} \wedge Y^{*}, X^{*} \vee Y^{*}, X^{*} \supset Y^{*}$, $\sim X^{*},\left(\exists x_{i}\right) X^{*},\left(\forall x_{i}\right) X^{*}$ we take $X \wedge Y, X \vee Y, X \supset Y, \sim X,\left(\exists x_{i}\right) X,\left(\forall x_{i}\right) X$ respectively, where $X^{*}, Y^{*}$ correspond to $X, Y$ respectively. Clearly, for any sentence $X^{*}$ we have $X^{*}$ holding in $\mathscr{L}^{*}$ if and only if the corresponding sentence $X$ holds in $\mathscr{L}$.

2. The main theorem, covering numbers, a characterisation of $h h$-simple sets. We require the notions of $h h$-simple set and maximal set to be relativised with respect to a given r.e. set in the following way. An r.e. set $\alpha$ is called $h h$-simple in its r.e. superset $\beta$ if $\beta-\alpha$ is infinite and if there is no recursive sequence of disjoint finite subsets of $\beta$ each of which intersects $\beta-\alpha$. Likewise, $\alpha$ is called maximal in its r.e. superset $\beta$ if $\beta-\alpha$ is infinite and if there is no r.e. set $\gamma$ such that $\gamma-\alpha, \beta-\gamma$ are both infinite and $\alpha \subseteq \gamma \subseteq \beta$. 
THEOREM 2. If $\alpha, \beta$ are any r.e. sets with $\alpha \subseteq \beta$ then either there exists a recursive set $\delta$ such that $\alpha \cup \delta=\beta$ or $\alpha$ is not hh-simple in $\beta$.

Proof. The method of proof is one which was first used in Friedberg [4] and later by Yates in [11].

Let $R$ be a ternary recursive predicate. Define the binary function $S$ by

$$
S(x, y)=\sup \left\{z \mid(E u)_{u \leqq x}(v)_{v \leqq z}(E w)_{w \leqq y} R(u, v, w)\right\} .
$$

Note that for some $x, y$ we may have $S(x, y)=\infty$, and that the relation $x>S(y, z)$ is recursive as a relation between $x, y, z$. Supposing $R$ is given we construct from it a recursive sequence $\left\{\delta_{i}\right\}$ of r.e. sets by simultaneous enumeration in steps $0,1, \ldots$ as follows. The sets $\alpha, \beta$ are to be effectively enumerated as the construction proceeds. Let $\alpha_{j}, \beta_{j}, \delta_{i, j}$ denote the finite sets of numbers which have been enumerated in $\alpha, \beta, \delta_{i}$ respectively by the end of step $j$.

Step 0. Do nothing.

Step $j+1$. Let $e$ be the least $i$ if any such that $\delta_{i, j}-\alpha_{j}$ is empty and such that $\beta_{j}$ contains a number $>S(i, j)$ which is not in $\alpha_{j} \cup\left\{\delta_{x, j} \mid x \geqq 0\right\}$. When $e$ exists, enumerate the least member of

$$
\left(\beta_{j} \cap\{x \mid x>S(i, j)\}\right)-\left(\alpha_{j} \cup \bigcup\left\{\delta_{x, j} \mid x \geqq 0\right\}\right)
$$

in $\delta_{e}$. Otherwise do nothing.

It is clear that this construction generates from any ternary recursive relation $R$ a recursive sequence $\left\{\delta_{i}\right\}$ of disjoint r.e. subsets of $\beta$.

LEMMA 1. If $(E x)(y)(E z) R(x, y, z)$ holds then $\bigcup\left\{\delta_{x} \mid x \geqq 0\right\}$ is recursive or there is a recursive subset $\delta$ of $\beta$ such that $\alpha \cup \delta=\beta$.

Proof. Let $e$ be the least number such that $(y)(E z) R(e, y, z)$ then from the definition of $S$ we have $\lim _{z} S(e, z)=\infty$. Also from the definition of $S, S(e, x)$ is increasing with $x$ and for any $y \geqq e$ we have $S(y, x) \geqq S(e, x)$. It follows that the set $\sigma=\bigcup\left\{\delta_{x} \mid x \geqq e\right\}$ is recursive, because according to the construction a number can only enter $\sigma$ after step $j$ if it is $>S(e, j)$. If $\beta-(\alpha \cup \sigma)$ is infinite then an infinite number of members of $\beta-\alpha$ become and remain available for enumeration in $\delta_{0}, \delta_{1}, \ldots, \delta_{e-1}$. Thus each of $\delta_{0}, \delta_{1}, \ldots, \delta_{e-1}$ will contain a member of $\beta-\alpha$ and so be finite, which implies that $\bigcup\left\{\delta_{x} \mid x \geqq 0\right\}$ is recursive. However, if $\beta-(\alpha \cup \sigma)$ is finite, setting $\delta=\sigma \cup(\beta-(\alpha \cup \sigma))$ we see that $\delta$ is recursive and that $\alpha \cup \delta=\beta$. This completes the proof of the lemma.

Returning to the proof of the theorem we now observe that given any disjoint recursive sequence $\left\{\varepsilon_{i}\right\}$ we can effectively find a ternary recursive relation $R$ such that

$$
(E x)(y)(E z) R(x, y, z) \Leftrightarrow \beta-\left(\alpha \cup \varepsilon_{0} \cup \varepsilon_{1} \cup \cdots\right) \text { is finite. }
$$

To see this we argue as follows. We can pass effectively from $\left\{\varepsilon_{i}\right\}$ to a strongly r.e. 
double sequence $\left\{\varepsilon_{i, j}\right\}$ of finite sets such that $\left\{\varepsilon_{i, j}\right\}$ is increasing in $j$ for fixed $i$ and such that for each $i, \lim _{x} \varepsilon_{i, x}=\varepsilon_{i}$. Now we have

$$
\begin{aligned}
\beta-\left(\alpha \cup \varepsilon_{0}\right. & \left.\cup \varepsilon_{1} \cup \cdots\right) \text { is finite } \\
& \Leftrightarrow(E x)(y)\left[y \leqq x \vee y \in\left(\alpha \cup \varepsilon_{0} \cup \varepsilon_{1} \cup \ldots\right) \vee y \notin \beta\right] . \\
& \Leftrightarrow(E x)(y)(z)(E w)\left[y \leqq x \vee y \in\left(\alpha_{w} \cup \varepsilon_{0, w} \cup \varepsilon_{1, w} \cup \cdots \cup \varepsilon_{w, w}\right) \vee y \notin \beta_{z}\right] .
\end{aligned}
$$

Let $P, Q$ be singularly recursive functions such that $(P(0), Q(0)),(P(1), Q(1)), \ldots$ is an enumeration of all ordered pairs of natural numbers. The last proposition can be written

$$
(E x)(y)(E z)\left[P(y) \leqq x \vee P(y) \in\left(\alpha_{z} \cup \varepsilon_{0, z} \cup \varepsilon_{1, z} \cup \cdots \cup \varepsilon_{z, z}\right) \vee P(y) \in \beta_{Q(y)}\right] .
$$

The relation within the square brackets is recursive and so will serve as $R(x, y, z)$.

Combining the two constructions we obtain an effective operation which maps any recursive disjoint sequence $\left\{\varepsilon_{i}\right\}$ into a recursive sequence $\left\{\delta_{i}\right\}$ of disjoint subsets of $\beta$. More precisely, if $E$ is the p.r. function defined by

$$
E(x)=y \Leftrightarrow x \in \varepsilon_{y}
$$

and $D$ is the corresponding p.r. function defined from $\left\{\delta_{i}\right\}$ then an index of $D$ can be found effectively from an index of $E$. By the recursion theorem [1, p. 176] we can choose $\left\{\varepsilon_{i}\right\}$ so that $\left\{\delta_{i}\right\}=\left\{\varepsilon_{i}\right\}$. For the rest of the proof suppose that $\left\{\varepsilon_{i}\right\}$ has been so chosen. There are now two possibilities. Firstly, we may have $(E x)(y)(E z) R(x, y, z)$ in which case from Lemma 1 either the theorem is immediate or we have $\bigcup\left\{\delta_{x} \mid x \geqq 0\right\}$ recursive. But from (8) we see that we also have $\beta-\left(\alpha \cup \delta_{0} \cup \delta_{1}\right.$ $\cup \ldots)$ finite. Thus whenever $(E x)(y)(E z) R(x, y, z)$ there is a recursive subset $\delta$ of $\beta$ such that $\alpha \cup \delta=\beta$. Secondly, we may have $(x)(E y)(z)[\neg R(x, y, z)]$ in which case it follows from (8) that $\beta-\left(\alpha \cup \delta_{0} \cup \delta_{1} \cup \ldots\right)$ is infinite. Looking back at the construction of $\left\{\delta_{i}\right\}$ from $R$ it is easy to see that in this case $\lim _{z} S(e, z)$ exists for all $e$. From this it follows that each $\delta_{i}$ contains a member of $\beta-\alpha$. For suppose $\delta_{e}$ did not intersect $\beta-\alpha$ then in the course of the construction we should enumerate in $\delta_{e}$ any member of $\beta-\alpha$ which is $>\lim _{z} S(e, z)$ and which is not in some other $\delta_{i}$. Thus if $\delta_{e}$ does not intersect $\beta-\alpha$ we have $\beta-\left(\alpha \cup \delta_{0} \cup \delta_{1} \cup \cdots\right)$ finite, which is impossible. This completes the proof of the theorem.

As a first application of the main theorem we prove a result which settles the first of the two claims of Myhill mentioned in the Introduction.

COROLlaRY 1. If the complement of an r.e. set $\alpha$ has covering number $n>0$ then $\alpha$ is the intersection of $n$ maximal sets $\mu_{1}, \ldots, \mu_{n}$ any two of which differ by an infinite set.

Proof. Suppose $\alpha$ is r.e. and that $\alpha^{\prime}$ has covering number $n$. From the definition of covering number there exist disjoint r.e. sets $\kappa_{1}, \ldots, \kappa_{n}$ such that each intersects $\alpha^{\prime}$ infinitely. Also, $\alpha$ must be $h h$-simple otherwise its covering number would be $\infty$. Taking $\beta=\alpha \cup \kappa_{1} \cup \cdots \cup \kappa_{n}$ in the theorem we see that there exists a recursive 
set $\delta$ such that $\alpha \cup \delta=\beta$. Thus $\alpha \cup \beta^{\prime}=\alpha \cup \delta^{\prime}$ is r.e. Enumerate $\beta$ and $\alpha \cup \beta^{\prime}$ simultaneously, and let

$$
\begin{aligned}
& \gamma_{0}=\left\{x \mid x \text { is enumerated in } \beta \text { before } \alpha \cup \beta^{\prime}\right\}, \\
& \gamma_{1}=\left\{x \mid x \text { is enumerated in } \alpha \cup \beta^{\prime} \text { before } \beta\right\} .
\end{aligned}
$$

Since $\beta, \alpha \cup \beta^{\prime}$ are disjoint in $\alpha^{\prime}$ we see that for $1 \leqq i \leqq n$ we have $\kappa_{i} \cap \gamma_{0} \cap \alpha^{\prime}$ $=\kappa_{i} \cap \alpha^{\prime}$ and $\gamma_{1} \cap \alpha^{\prime}=\beta^{\prime}$. Thus if $\beta^{\prime}$ were infinite we should have $n+1$ disjoint r.e. sets, namely $\kappa_{1} \cap \gamma_{0}, \ldots, \kappa_{n} \cap \gamma_{0}$ and $\gamma_{1}$, which intersect $\alpha^{\prime}$ infinitely. It follows that $\beta^{\prime}$ is finite, and so we may assume $\beta=\nu$. For $1 \leqq i \leqq n$ let

$$
\mu_{i}=\alpha \cup \bigcup\left\{\kappa_{x} \mid 1 \leqq x \leqq n \& x \neq i\right\} .
$$

It is clear that any two of $\mu_{1}, \ldots, \mu_{n}$ differ by an infinite set; we shall show that $\mu_{1}, \ldots, \mu_{n}$ are all maximal. It suffices to consider $\mu_{1}$ which is plainly coinfinite. Now suppose $\beta$ (not the same $\beta$ as before) is an r.e. set such that $\mu_{1} \subseteq \beta \subseteq \nu$. We form $\delta_{0}, \delta_{1}$ from $\alpha, \beta$ just as we formed $\gamma_{0}, \gamma_{1}$ above; thus $\kappa_{1} \cap \delta_{0}, \ldots, \kappa_{n} \cap \delta_{0}$ and $\delta_{1}$ are disjoint r.e. sets. Further, since $\beta \supseteq \mu_{1}$ we know that $\kappa_{2} \cap \delta_{0}, \ldots, \kappa_{n} \cap \delta_{0}$ all intersect $\alpha^{\prime}$ infinitely. Hence either $\kappa_{1} \cap \delta_{0} \cap \alpha^{\prime}$ is finite or $\delta_{1} \cap \alpha^{\prime}$ is finite. But $\beta \cap \kappa_{1} \cap \alpha^{\prime}=\kappa_{1} \cap \delta_{0} \cap \alpha^{\prime}$ and $\beta^{\prime}=\delta_{1} \cap \alpha^{\prime}$. Thus in one case $\beta-\mu_{1}$ is finite and in the other $\beta^{\prime}$ is finite. Hence $\mu_{1}$ is maximal, and similarly so are $\mu_{2}, \ldots, \mu_{n}$. This completes the proof.

This corollary is just the converse of the result of Yates mentioned in the Introduction. From Yates's theorem we see that when $\alpha^{\prime}$ has covering number $n$ the maximal sets $\mu_{1}, \ldots, \mu_{n}$ are determined to within finite sets by $\alpha$.

We can extract slightly more from the argument used to prove the corollary. Define the partition number of a set $\chi$ to be

sup \{cardinality of $\mathfrak{A} \mid \mathfrak{A}$ is a class of disjoint recursive sets each intersecting $\chi$ infinitely and such that $\bigcup \mathfrak{A}=\nu\}$.

Suppose that $\alpha$ is $h h$-simple and note that $\kappa_{1} \cap \gamma_{0}, \ldots, \kappa_{n} \cap \gamma_{0}, \gamma_{1}$ are recursive in the first part of the proof since they are disjoint r.e. sets which exhaust $\nu$. This shows that the partition number of $\alpha^{\prime}$ is $\geqq$ the covering number of $\alpha^{\prime}$, and so for $h h$-simple sets the covering number and partition number of the complement are the same. Now let $\rho$ be any recursive set such that $\rho \cap \alpha^{\prime}$ is infinite where $\alpha$ is still $h h$-simple. Taking $\rho=\kappa_{1}$ in the second part of the proof we see that either $\alpha \cap \rho^{\prime}$ is maximal or we can split $\rho$ into disjoint recursive sets $\rho \cap \delta_{0}, \rho \cap \delta_{1}$ each having infinite intersection with $\alpha^{\prime}$. Notice also that if the partition number of $\rho \cap \alpha^{\prime}$ is $\infty$ then so is the partition number of either $\rho \cap \delta_{0} \cap \alpha^{\prime}$ or $\rho \cap \delta_{1} \cap \alpha^{\prime}$. Thus if $\alpha$ is $h h$-simple and $\alpha^{\prime}$ has partition number $\infty$ we can form two sequences of recursive sets $\left\{\rho_{i}\right\},\left\{\sigma_{i}\right\}$ such that

$$
\rho_{0} \cup \sigma_{0}=\nu, \quad \rho_{0} \cap \sigma_{0}=\varnothing,
$$


and for all $i: \rho_{i}-\alpha$ has covering number $\infty, \sigma_{i}-\alpha$ is infinite,

$$
\rho_{i+1} \cup \sigma_{i+1}=\rho_{i}, \quad \rho_{i+1} \cap \sigma_{i+1}=\varnothing .
$$

Now define

$$
\tau_{i}=\left(\sigma_{i}-\{0,1, \ldots, i-1\}\right) \cup\left(\{i\}-\left(\sigma_{0} \cup \sigma_{1} \cup \ldots \cup \sigma_{i-1}\right)\right)
$$

and we see that $\left\{\tau_{i}\right\}$ is a sequence of disjoint recursive sets exhausting $\nu$ and such that each member of the class intersects $\alpha^{\prime}$ infinitely.

As a second application of the main theorem we give a characterisation of $h$-simple sets.

THEOREM 3. An r.e. set $\alpha$ is hh-simple if and only if it is coinfinite and for any r.e. set $\beta$ the set $\alpha \cup \beta^{\prime}$ is r.e.

Proof. For the only if part we may assume that $\beta$ is an r.e. superset of $\alpha$ because $\alpha \cup \beta^{\prime}=\alpha \cup(\alpha \cup \beta)^{\prime}$. But if $\alpha$ is $h$-simple then it is $h h$-simple in any superset $\beta$. Thus from Theorem 2 there exists a recursive subset $\delta$ of $\beta$ such that $\alpha \cup \delta=\beta$. Since $\alpha \cup \delta^{\prime}=\alpha \cup \beta^{\prime}$ we have the result.

For the if part, supposing $\alpha$ is an r.e. coinfinite but non-hh-simple set we shall construct an r.e. superset $\beta$ of $\alpha$ such that $\alpha \cup \beta^{\prime}$ is not r.e. Accordingly, let $\left\{\delta_{i}\right\}$ be any recursive sequence of finite sets each of which intersects $\alpha^{\prime}$, and let $\left\{\omega_{i}\right\}$ be any recursive enumeration of all r.e. sets. We let $\beta$ be

$$
\alpha \cup \bigcup\left\{\delta_{x} \cap \omega_{x} \mid x \geqq 0\right\} .
$$

If $\alpha \cup \beta^{\prime}$ is r.e. then it is $\omega_{e}$ for some $e$. Now let $f$ be a member of $\delta_{e} \cap \alpha^{\prime}$, then from the definition of $\beta$

$$
f \in \omega_{e} \Leftrightarrow f \in \beta .
$$

But from the equation $\alpha \cup \beta^{\prime}=\omega_{e}$ we infer the opposite. Thus $\alpha \cup \beta^{\prime}$ is not r.e.

Corollary. If $\alpha_{1}, \alpha_{2}$ are both hh-simple then so is $\alpha_{1} \cap \alpha_{2}$.

Proof. From the theorem it is sufficient to show that if $\beta$ is any r.e. set then $\left(\alpha_{1} \cap \alpha_{2}\right) \cup \beta^{\prime}$ is r.e. But we have $\alpha_{1} \cup \beta^{\prime}, \alpha_{2} \cup \beta^{\prime}$ r.e. because $\alpha_{1}, \alpha_{2}$ are $h h$-simple respectively, and

$$
\left(\alpha_{1} \cup \beta^{\prime}\right) \cap\left(\alpha_{2} \cup \beta^{\prime}\right)=\left(\alpha_{1} \cap \alpha_{2}\right) \cup \beta^{\prime} .
$$

The set on the left is r.e. being the intersection of two r.e. sets. This completes the proof.

To complete the section we generalise the method of Theorem 2. R. W. Robinson has noticed that the use of the recursion theorem in the proof of Theorem 2 can be eliminated. We use his observation in what follows although we could equally well use the recursion theorem again. First we give some definitions and lemmas.

A set $\alpha$ is called hh-immune if it infinite and there is no recursive sequence of disjoint finite sets each member of which intersects $\alpha$. 
Let $\boldsymbol{\omega}=\left(\omega_{1}, \ldots, \omega_{k}\right)$ be any $k$-tuple of r.e. sets. The order of $\omega$ denoted by $|\omega|$ is defined to be the number of subsets $\iota$ of $\{1,2, \ldots, k\}=\kappa$ for which $\omega(\iota)$ is infinite. We say that the ordered pair $\left(\boldsymbol{\omega}^{0}, \boldsymbol{\omega}^{1}\right)$ of $k$-tuples of r.e. sets is a splitting of $\boldsymbol{\omega}$ if there exist disjoint complementary recursive sets $\delta_{0}, \delta_{1}$ and (1-1) recursive functions $D_{0}, D_{1}$ such that $D_{0}(v)=\delta_{0}, D_{1}(v)=\delta_{1}$, and such that $\omega_{i}^{0}=D_{0}^{-1}\left(\omega_{i}\right), \omega_{i}^{1}=D_{1}^{-1}\left(\omega_{i}\right)$ for $1 \leqq i \leqq k$.

LEMMA 2. If $\omega(\imath)$ is infinite and non-hh-immune and if $\left(\omega^{0}, \omega^{1}\right)$ is a splitting of the $k$-tuple of r.e. sets $\omega$ then one of $\omega^{0}(\iota), \omega^{1}(\iota)$ is both infinite and non-hh-immune.

Proof. It is sufficient to show that one of $\omega(\iota) \cap \delta_{0}, \omega(\iota) \cap \delta_{1}$ is both infinite and non- $h h$-immune. This is because $\omega^{0}(\iota)=D_{0}^{-1}\left(\omega(\iota) \cap \delta_{0}\right)$ and $\omega^{1}(\iota)=D_{1}^{-1}\left(\omega(\iota) \cap \delta_{1}\right)$. If one of $\omega(\iota) \cap \delta_{0}, \omega(\iota) \cap \delta_{1}$ is finite there is nothing to prove. Suppose for reductio ad absurdum that both sets are $h h$-immune. From its definition $\omega(\iota)$ has the form $\beta-\alpha$ where $\alpha$ is an r.e. subset of the r.e. set $\beta$. Since $\omega(\iota) \cap \delta_{1}$ is $h h$-immune $\delta_{0} \cup \alpha$ is $h h$-simple in $\delta_{0} \cup \beta$. From Theorem 2 we see that $\delta_{0} \cup \beta^{\prime}$ is r.e. and so $\alpha \cup \delta_{0} \cup \beta^{\prime}$ is $h$ h-simple since it is r.e. and has complement $\omega(\iota) \cap \delta_{1}$. Similarly $\alpha \cup \delta_{1} \cup \beta^{\prime}$ is $h h$-simple. Now $\alpha \cup \beta^{\prime}=\left(\alpha \cup \delta_{0} \cup \beta^{\prime}\right) \cap\left(\alpha \cup \delta_{1} \cup \beta^{\prime}\right)$ is $h h$-simple by the Corollary of Theorem 3, and so $\omega(\iota)$ is $h h$-immune. This proves the lemma.

LEMMA 3. If $\omega=\left(\omega_{1}, \ldots, \omega_{k}\right)$ is a $k$-tuple of r.e. sets and none of $\omega_{1}^{\prime}, \ldots, \omega_{k}^{\prime}$ is finite and $\omega(\varnothing)$ is finite then there exists a splitting $\left(\omega^{0}, \omega^{1}\right)$ of $\omega$ such that $\left|\omega^{0}\right|<|\omega|$ and $\left|\omega^{1}\right|<|\omega|$.

Proof. Let $\lambda$ be a subclass of $\kappa$ such that $\bigcup\left\{\omega_{x} \mid x \in \lambda\right\}$ is cofinite and is minimal with respect to inclusion. Let $p$ be any member of $\lambda$. We enumerate $\omega_{p}$ and $\chi=\bigcup\left\{\omega_{x} \mid x \in \lambda-\{p\}\right\}$ simultaneously, and let

$$
\begin{aligned}
& \delta_{0}=\left\{y \mid y \text { is enumerated in } \omega_{p} \text { before } \chi\right\}, \\
& \delta_{1}=\left\{y \mid y \text { is enumerated in } \chi \text { before } \omega_{p}\right\} .
\end{aligned}
$$

Then $\delta_{0}, \delta_{1}$ are disjoint r.e. sets whose union is $\omega_{p} \cup \chi$ and therefore cofinite. We may suppose since we are only interested in infinite sets that $\delta_{0} \cup \delta_{1}=\nu$. Hence there exist (1-1) recursive functions $D_{0}, D_{1}$ such that $D_{0}(v)=\delta_{0}, D_{1}(v)=\delta_{1}$. Let $\left(\omega^{0}, \omega^{1}\right)$ be the corresponding splitting of $\omega$. Now $\omega^{0}(\iota)$, or $\omega^{1}(\iota)$ can only be infinite if $\omega(\iota)$ is infinite. Also $\omega^{0}(\iota)$ will not be infinite unless $\iota$ contains $p$, although $\omega(\iota)$ will be infinite for some $\iota$ not containing $p$ because $\omega_{p}^{\prime}$ is infinite. Also $\omega^{1}(\iota)$ will not be infinite for any $\iota$ disjoint from $\lambda-\{p\}$, although $\omega(\iota)$ will be infinite for some such $\iota$ because $\bigcap\left\{\omega_{x}^{\prime} \mid x \in \lambda-\{p\}\right\}$ is infinite. Thus $\left|\omega^{0}\right|<|\omega|$ and $\left|\omega^{1}\right|<|\omega|$ as alleged.

THEOREM 4. Let $\omega=\left(\omega_{1}, \ldots, \omega_{k}\right)$ be a $k$-tuple of r.e. sets and let

$$
\mathscr{K} \subseteq\{\iota \mid \iota \subseteq \kappa \& \omega(\iota) \text { is not hh-immune or finite }\},
$$

then there exists a disjoint recursive sequence $\left\{\delta_{i}^{\iota} \mid \iota \in \mathscr{K} \quad \& \quad i \geqq 0\right\}$ of finite sets such that $\delta_{i}^{\iota}$ intersects $\omega(\iota)$ for all $\iota$ in $\mathscr{K}$ and all $i \geqq 0$. 
Proof. Take any effective (1-1) map of $\mathscr{K} \times \nu$ onto $\nu$ and denote by $\leqq$ the wellordering of $\mathscr{K} \times \nu$ induced by the natural ordering of $\nu$. Without loss of generality we may suppose that all of $\omega_{1}^{\prime}, \ldots, \omega_{k}^{\prime}$ are infinite, because if $\omega^{*}$ comes from $\omega$ by removal of a cofinite set then modulo finite sets the infinite sets of the form $\omega(\iota)$ are the same as those of the form $\omega^{*}(\iota)$. If $\omega(\varnothing)$ is finite we obtain a splitting $\left(\omega^{0}, \omega^{1}\right)$ of $\omega$ such that $\omega^{0}, \omega^{1}$ both have order < the order of $\omega$ from Lemma 3. For $r=0,1$ define

$$
\mathscr{K}^{r}=\left\{\imath \mid \iota \in \mathscr{K} \& \omega^{r}(\iota) \text { is not } h h \text {-immune or finite }\right\} .
$$

From Lemma 2 we have $\mathscr{K}^{0} \cup \mathscr{K}^{1}=\mathscr{K}$ and so if the theorem holds for $\omega^{0}$ and for $\omega^{1}$ it also holds for $\omega$.

Supposing now that $\omega_{1}^{\prime}, \ldots, \omega_{k}^{\prime}$ are all infinite and that $\omega(\varnothing)$ is infinite we attempt to construct the required sequence of disjoint finite sets by simultaneous enumeration in steps $0,1, \ldots$ For $\iota$ in $\mathscr{K}$ let $\delta_{i, j}^{\iota}$ be the finite set of numbers which have been enumerated in $\delta_{i}^{l}$ by the end of step $j$. For $1 \leqq i \leqq k$ let $\omega_{i, j}$ be the finite set of numbers which have been enumerated in $\omega_{i}$ by the end of step $j$. If $\varnothing$ is in $\mathscr{K}$ then we let $\omega$ be an r.e. superset of $\omega(\varnothing)^{\prime}$ such that $\omega(\varnothing)^{\prime} \cup \omega^{\prime}$ is not r.e.; such an $\omega$ exists by the last theorem. Otherwise we let $\omega$ be $\omega(\varnothing)^{\prime}$. We let $\omega_{j}$ be the finite set of numbers which have been enumerated in $\omega$ by the end of step $j$. For $\iota \neq \varnothing$ we define $\omega_{j}(\iota)$ to be

$$
\bigcap\left\{\omega_{x, j} \mid x \in \iota\right\} \cap \bigcap\left\{\omega_{x, j}^{\prime} \mid x \in \kappa-\iota\right\}
$$

we define $\omega_{j}(\varnothing)$ to be

$$
\omega_{j} \cap \bigcap\left\{\omega_{x, j}^{\prime} \mid x \in \kappa\right\} .
$$

In the course of the construction for each $\iota$ in $\mathscr{K}$ we define a binary recursive function $S^{\imath}$ as follows:

$$
\begin{aligned}
& S^{\iota}(x, 0)=0, \\
S^{\iota}(x, j+1)= & S^{\iota}(x, j) \text { if } \delta_{x, j}^{\iota} \text { intersects } \omega_{j}(\iota), \\
= & S^{\iota}(x, j)+1 \text { otherwise. }
\end{aligned}
$$

Step 0. Do nothing.

Step $j+1$. Let $(\varepsilon, e)$ be the least member of $\mathscr{K} \times \nu$ such that $\delta_{e, j}^{\varepsilon} \cap \omega_{j}(\varepsilon)$ is empty and such that $\omega_{j+1}(\varepsilon)$ contains a number $f>\max \left\{S^{\varepsilon}(x, j) \mid(\iota, x)<(\varepsilon, e)\right\}$ which is not in $\bigcup\left\{\delta_{x, j}^{\iota} \dagger \iota \in \mathscr{K} \quad \& \quad x \geqq 0\right\}$. When $(\varepsilon, e)$ exists enumerate the least such $f$ in $\delta_{e}^{\varepsilon}$. Otherwise do nothing.

It is easy to check that the construction is effective and also that, if $\delta_{i}^{\iota}$ intersects $\omega(\iota)$, then $\delta_{i}^{\iota}$ is finite and $\lim _{y} S^{\iota}(i, y)$ exists. It is also easy to see from step $j+1$ that the sequence $\left\{\delta_{i}^{\iota} \mid \iota \in \mathscr{K} \quad \& \quad i \geqq 0\right\}$ is disjoint, i.e., $\delta_{i}^{\iota}$ intersects $\delta_{h}^{\eta}$ only if $\iota=\eta$ and $i=h$. Thus there is nothing to prove unless $\delta_{i}$ fails to intersect $\omega(\iota)$ for some pair $(\iota, i)$. Let $(\varepsilon, e)$ be the least such pair; then $S^{\varepsilon}(e, y)$ increases as $y$ increases and has limit $\infty$. Hence, because of the clause $f>\max \left\{S_{\iota}(x, j) \mid(\iota, x)<(\varepsilon, e)\right\}$ in step $j+1$, we see that $\bigcup\left\{\delta_{i}^{l} \mid(\iota, i)>(\varepsilon, e)\right\}$ is recursive. Also, by choice of $(\varepsilon, e)$ for $(\iota, i)<(\varepsilon, e)$ we have that $\delta_{i}^{\iota}$ is finite and $\lim _{y} S_{\iota}(i, y)$ exists. If $\varepsilon=\varnothing$ then $\bigcup\left\{\delta_{i}^{\iota} \mid(\iota, i)>(\varepsilon, e)\right\}$ 
covers $\omega \cap \omega(\varnothing)$ except possibly for a finite number of numbers, otherwise a member of $\omega \cap \omega(\varnothing)$ would eventually be enumerated in $\delta_{e}^{\varepsilon}$. However, this implies that $\omega(\varnothing)^{\prime} \cup \omega^{\prime}$ is r.e. which is contrary to the choice of $\omega$; thus $\varepsilon \neq \varnothing$. Now $\bigcup\left\{\delta_{i}^{\imath} \mid(\iota, i)>(\varepsilon, e)\right\}$ covers $\omega(\varepsilon)$ except possibly for a finite number of numbers, otherwise a member of $\omega(\varepsilon)$ would eventually be enumerated in $\delta_{e}^{\varepsilon}$. It follows that there is a recursive set $\delta$ such that $\omega(\varepsilon) \subseteq \delta \subseteq \omega$. Now take $\delta_{0}=\delta$ and $\delta_{1}=\delta^{\prime}$, and let $\left(\omega^{0}, \omega^{1}\right)$ be any corresponding splitting of $\omega$. We define

$$
\begin{aligned}
& \mathscr{K}^{0}=\left\{\imath \mid \iota \in \mathscr{K} \quad \& \quad \iota \neq \varnothing \& \omega^{0}(\iota) \text { is not } h h \text {-immune or finite }\right\}, \\
& \mathscr{K}^{1}=\left\{\iota \mid \iota \in \mathscr{K} \quad \& \omega^{1}(\iota) \text { is not } h h \text {-immune or finite }\right\} .
\end{aligned}
$$

If $\varnothing$ is not in $\mathscr{K}$ then it is clear that $\mathscr{K}^{0} \cup \mathscr{K}^{1}=\mathscr{K}$ from Lemma 2 . If $\varnothing$ is in $\mathscr{K}$ we have $\omega^{\prime} \cup\left(\delta_{1} \cap \omega(\varnothing)^{\prime}\right)$ not r.e., because

$$
\omega^{\prime} \cup\left(\delta_{1} \cap \omega(\varnothing)^{\prime}\right) \cup \omega(\varnothing)^{\prime}=\omega^{\prime} \cup \omega(\varnothing)^{\prime}
$$

and the set on the right is not r.e. by choice of $\omega$. Hence in this case $\omega(\varnothing) \cap \delta_{1}$ is not finite or $h$-immune, from which it follows that $\omega^{1}(\varnothing)$ is not finite or $h h$ immune. Thus in any case we get $\mathscr{K}^{0} \cup \mathscr{K}^{1}=\mathscr{K}$. The theorem will hold for the pair $(\mathscr{K}, \omega)$ if it holds for both the pairs $\left(\mathscr{K}^{0}, \omega^{0}\right),\left(\mathscr{K}^{1}, \omega^{1}\right)$. Since $\omega(\varepsilon) \subseteq \delta=\delta_{0}$ we have $\omega^{1}(\varepsilon)$ empty, and so $\left|\omega^{1}\right|<|\omega|$. If $\varnothing$ is not in $\mathscr{K}$ then from $\delta \subseteq \omega$ we have $\boldsymbol{\omega}^{0}(\varnothing)$ empty, and so $\left|\boldsymbol{\omega}^{0}\right|<|\boldsymbol{\omega}|$. If $\varnothing$ is in $\mathscr{K}$ then since $\varnothing$ is not in $\mathscr{K}^{0}$ the cardinality of $\mathscr{K}^{0}$ is less than the cardinality of $\mathscr{K}$. The theorem now follows by induction on

$$
|\omega|+\text { cardinality of } \mathscr{K} \text {. }
$$

The starting case is that in which the cardinality of $\mathscr{K}$ is 1 ; the theorem is obvious in this case.

3. An $h h$-simple set with no maximal superset. The construction of this section settles the second claim of Myhill mentioned in the Introduction.

Let $\left\{\chi_{i}\right\}$ be a recursive enumeration of all r.e. sets, recursive in the sense that $x \in \chi_{y}$ is a binary r.e. relation. Imagine a clerk simultaneously enumerating all the sets of the sequence. At each step the clerk enumerates a pair $(x, y)$, and the set of all pairs which he enumerates is $\left\{(x, y) \mid x \in \chi_{y}\right\}$. For technical reasons each pair which is enumerated at all is enumerated at an infinite number of steps.

Let $\mathfrak{a}, \mathfrak{b}, \ldots$, denote finite strings of 0 's and 1's possibly empty, and denote the set of all such strings by $\mathfrak{L}$. We consider (1-1) functions from $\mathfrak{L}$ into $\nu$ which we call number trees. The members of a number tree are the members of its range. We denote by $\mathfrak{a} * \mathfrak{b}$ the string formed by concatenating $\mathfrak{b}$ to the right of $\mathfrak{a}$. If $T$ is a number tree and $\mathfrak{a}$ is any member of $\mathfrak{Q}$, then $T[\mathfrak{a}]$ denotes the number tree defined by $T[\mathfrak{a}](\mathfrak{x})=T(\mathfrak{a} * \mathfrak{x})$. We order $\mathfrak{L}$ linearly as follows: $\varnothing, 0,1,00,01,10,11,000$, $\ldots$, that is to say first by length and then lexicographically. When we speak of the least member of a subset $\mathfrak{D}$ of $\mathfrak{Q}$ we mean the member of $\mathfrak{D}$ which occurs earliest in this sequence; if $\mathfrak{a}$ occurs before $\mathfrak{b}$ we write $\mathfrak{a}<\mathfrak{b}$. Also, the members of a number 
tree $T$ have a linear order $\prec_{T}$ (abbreviated to $\prec$ whenever there is no doubt which $T$ is meant) induced by the ordering of $\mathfrak{L}$ :

$$
x \prec y \Leftrightarrow(E \mathfrak{a})(E \mathfrak{b})[x=T(\mathfrak{a}) \& y=T(\mathfrak{b}) \& \mathfrak{a}<\mathfrak{b}] .
$$

The construction which follows consists of steps $0,1, \ldots$ In each step $s$ we define a number tree $T_{s}$. Let $\eta_{s}$ consist of those numbers not in the range of $T_{s}$, then $\left\{\eta_{i}\right\}$ will be an increasing strongly r.e. sequence whose limit is the r.e. set required.

Let $\omega_{y, s}$ be the finite set defined as follows. Firstly, $\omega_{y, 0}=\varnothing$ for all $y$. Secondly, if $(x, y)$ is the pair which the clerk enumerates at step $s$ we set

$$
\begin{aligned}
\omega_{z, s+1} & =\omega_{y, s} \cup\{x\} \text { if } z=y \text { and } x \notin \omega_{y, s}, \\
& =\omega_{z, s}-\{x\} \text { if } z>y \text { and } x \notin \omega_{y, s}, \\
& =\omega_{z, s} \text { otherwise. }
\end{aligned}
$$

LeMma 4. For all $y, \lim _{z} \omega_{y, z}=\chi_{y}$.

Proof. Suppose that the lemma holds for all $y<e$. By inspection $\omega_{e, s} \subseteq \chi_{e}$. Let $m$ be any number in $\chi_{e}$ then by the induction hypothesis there exists $q$ such that for all $y<e$ and all $s>q$ we have $m$ not in $\omega_{y, s+1}-\omega_{y, s}$. For some $s \geqq q$ we have $m$ in $\omega_{e, s}$, because the clerk enumerates every pair an infinite number of times if at all. Also, from the last definition we see that $m$ is in $\omega_{e, t}-\omega_{e, t+1}$ for some $t \geqq s$ only if there exists $y<e$ such that $m$ is in $\omega_{y, t+1}-\omega_{y, t}$ which is impossible by choice of $q$. Therefore $m$ is in $\omega_{e, s}$ for all sufficiently large $s$ and the lemma is proved.

We define an auxiliary function $W$ by

$$
W(x, y, s)=\sum\left\{2^{y-z} \mid x \in \omega_{z, s} \& z \leqq y\right\} .
$$

It is clear from Lemma 4 that

$$
\lim _{w} W(x, y, w)=\sum\left\{2^{y-z} \mid x \in \chi_{z} \& z \leqq y\right\} .
$$

Step 0. Define $T_{0}$ by $T_{0}(\varnothing)=0$, and

$$
\begin{aligned}
& T_{0}(\mathfrak{x} * 0)=2 T_{0}(\mathfrak{x})+1, \\
& T_{0}(\mathfrak{x} * 1)=2 T_{0}(\mathfrak{x})+2 .
\end{aligned}
$$

It is easily verified that $T_{0}$ is a number tree onto $\nu$.

Step $s+1$. Let $(x, y)$ be the pair which the clerk enumerates at step $s$. If $x$ is not a member of $T_{s}$ or if $x=T_{s}(\mathfrak{x})$ and $\mathfrak{x}$ has length $\leqq y$ or if $x$ is in $\omega_{y, s}$ then do nothing. Otherwise let $\mathfrak{x}_{0}$ be the string of length $y$ of which $\mathfrak{x}$ is an extension. Let $\mathfrak{x}_{1}$ be the least extension of $\mathfrak{x}_{0}$ if any such that $\mathfrak{x}_{1}<\mathfrak{x}$ and such that $W\left(x_{1}, y, s\right)$ $<W(x, y, s+1)$ where $x_{1}=T\left(\mathfrak{x}_{1}\right)$. Define

$$
\begin{aligned}
T_{s+1}(\mathfrak{a}) & =x \text { if } \mathfrak{a}=\mathfrak{x}_{1}, \\
& =T_{s}(\mathfrak{a} * 0) \text { if } \mathfrak{a} \text { is one of } \mathfrak{x}, \mathfrak{x} * 0, \mathfrak{x} * 00, \ldots, \\
& =T_{s}(\mathfrak{a}) \text { otherwise. }
\end{aligned}
$$


We note that the range of $T_{s+1}$ is the range of $T_{s}$ with $x_{1}$ removed. If there is no $\mathfrak{x}_{1}$ satisfying the given conditions or if the step is vacuous anyway, we set $T_{s+1}=T_{s}$.

The construction which we have described is clearly effective. Given $i$ we can effectively enumerate $\eta_{i}$ and can effectively find its cardinality. Further, by inspection of step $s+1$ we see that $\eta_{s} \subseteq \eta_{s+1}$. Hence $\left\{\eta_{i}\right\}$ being a strongly r.e. increasing sequence has an r.e. limit which we denote by $\eta$.

LEMMA $5 . \lim _{x} T_{x}$ exists.

Proof. For reductio ad absurdum let $\mathfrak{b}$ be the least string such that $\lim _{x} T_{x}(\mathfrak{b})$ does not exist, then there are an infinite number of steps $s+1$ for which $T_{s}(\mathfrak{b})$ $\neq T_{s+1}(\mathfrak{b})$. For all sufficiently large $s$ such that $T_{s}(\mathfrak{b}) \neq T_{s+1}(\mathfrak{b})$ we have $\mathfrak{x}_{1}=\mathfrak{b}$; this is because at any step $s+1$ for which $T_{s} \neq T_{s+1}, \mathfrak{x}_{1}$ is the least string on which $T_{s+1}$ disagrees with $T_{s}$. At any step $s+1$ where $\mathfrak{x}_{1}=\mathfrak{b}$ the length $y$ of $\mathfrak{x}_{0}$ is $\leqq$ the length of $\mathfrak{b}$. Also, if $\mathfrak{x}_{1}=\mathfrak{b}$ at step $s+1$, then $W\left(T_{s}(\mathfrak{b}), y, s\right)<W\left(T_{s+1}(\mathfrak{b}), y, s+1\right)$. Let $y_{0}$ be the least number such that there are infinitely many steps $s+1$ with $\mathfrak{x}_{1}$ $=\mathfrak{b}$ and $y=y_{0}$. Then for all sufficiently large $s$ we have $W\left(T_{s}(\mathfrak{b}), y_{0}, s\right) \leqq W\left(T_{s+1}(\mathfrak{b})\right.$, $\left.y_{0}, s+1\right)$ either because $\mathfrak{x}_{1}$ is not defined, or because $\mathfrak{x}_{1}>\mathfrak{b}$, or because $\mathfrak{x}_{1}=\mathfrak{b}$, and $y \geqq y_{0}$. Further, for infinitely many $s$ the inequality is strict because there are infinitely many $s$ such that $\mathfrak{x}_{1}=\mathfrak{b}$ and $y=y_{0}$ at step $s+1$. This contradicts the inequality $W\left(T_{s}(\mathfrak{b}), y_{0}, s\right)<2^{y_{0}+1}$ which follows directly from the definition of $W$. This completes the proof of the lemma.

Let $T$ denote $\lim _{x} T_{x}$. Let $W(x, y)$ denote $\lim _{z} W(x, y, z)$.

LEMMA 6. Let e be a string of length e. Let $b=T(\mathfrak{b})$ where $\mathfrak{b}$ is an extension of $\mathrm{e}$. Let $t$ be the greatest number such that the pair enumerated at step $t$ is $(b, e)$ and such that $b$ is not in $\omega_{e, t}$. Then $T_{t}^{-1}(b)$ is an extension of $e$ and $W(b, e, t+1)=W(b, e)$.

Proof. If $t$ does not exist the conclusion is meant to be vacuous. We first notice that for any member $b$ of $T$ the length of $T_{s}^{-1}(b)$ is decreasing. Thus if $T^{-1}(b)$ has length $\geqq e$ so does $T_{s}^{-1}(b)$ for all $s$. Therefore there exists some string $\mathrm{e}^{*}$ of length $e$ of which $T_{t}^{-1}(b)$ is an extension. Let $s+1$ be the least step $>t$ if any such that $T_{s+1}^{-1}(b)$ is not an extension of $e^{*}$. Then we see that $\mathfrak{x}_{0}$ in step $s+1$ has length $y<e$ and that $x=b$. Thus the pair $(x, y)$ enumerated by the clerk at step $s$ has $x=b$ and $y<e$. Hence $b$ is not in $\omega_{e, s+1}$ which contradicts the choice of $t$. Thus no such step $s+1$ exists and $T_{t}^{-1}(b)$ is therefore an extension of $e$.

If $W(b, e, t+1) \neq W(b, e)$, this can only be because there exists $m \leqq e$ such that $b \in \chi_{m}-\omega_{m, t+1}$. It is clear that in fact $m<e$. Letting $s$ be the least number $>t$ such that the clerk enumerates $(b, m)$ at step $s$ we have the same contradiction as before.

LEMMA 7. Let e be a string of length $e$; then there exists $w$ such that for all but a finite number of extensions $\mathfrak{x}$ of $\mathrm{e}$ we have $W(T(\mathfrak{x}), e)=w$. 
Proof. Let $e^{-}$be the string of length $e-1$ of which $e$ is an extension, then we may assume that there exists $w^{-}$such that for all but a finite number of extensions $\mathfrak{x}$ of $\mathrm{e}^{-}$we have $W(T(\mathfrak{x}), e-1)=w^{-}$. From the definition of $W$ we see that for all but a finite number of extensions $x$ of $e$ we have

$$
2 w^{-} \leqq W(T(\mathfrak{x}), e) \leqq 2 w^{-}+1 .
$$

We may suppose that $W(T(\mathfrak{x}), e)=2 w^{-}+1$ for infinitely many extensions $\mathfrak{x}$ of $\mathrm{e}$, otherwise there is nothing to prove. Let $a$ be any extension of $e$ and let $a$ be such that for all $x \geqq a$ and for all $\mathfrak{x} \leqq \mathfrak{a}$ we have $T_{x}(\mathfrak{x})=T(\mathfrak{x})$. There are an infinite number of members $b$ of $T[e]$ for which $W(b, e)=2 w^{-}+1$. Each such $b$ is in $\chi_{e}$ and thus has associated with it a greatest $t$ such that $b$ is not in $\omega_{e, t}$ and such that $(b, e)$ is enumerated at step $t$. Since there are infinitely many such $b$ we can find one with $T_{t}^{-1}(b)=\mathfrak{b}>\mathfrak{a}$ and $t \geqq a$. From the last lemma $\mathfrak{b}$ is an extension of $e$ and $W(b, e, t+1)$ $=W(b, e)=2 w^{-}+1$. Since $T_{t+1}(\mathfrak{x})=T_{t}(\mathfrak{x})$ for all $\mathfrak{x} \leqq \mathfrak{a}$ by choice of $a$, we have $W(T(\mathfrak{a}), e, t) \geqq W(b, e, t+1)$. Hence $W(T(\mathfrak{a}), e) \geqq 2 w^{-}+1$. But $\mathfrak{a}$ is any extension of e and so $W(T(\mathfrak{x}), e)=2 w^{-}+1$ for all but a finite number of extensions $\mathfrak{x}$ of $\mathrm{e}$. This completes the proof.

LEMMA 8. For any string e the set $\eta \cup$ range $(T[\mathrm{e}])$ is r.e.

Proof. Let $\mathrm{e}$ have length $e$. From Lemma 7 there exist $w, \mathrm{e}_{0}$ such that $\mathrm{e}_{0}$ is an extension of $e$ and such that for all extensions $x$ of $e$ which are $\geqq e_{0}$ we have $W(T(\mathfrak{x}), e)=w$. Let $s_{0}$ be such that $T_{s}(\mathfrak{x})=T(\mathfrak{x})$ for all $s \geqq s_{0}$ and all $\mathfrak{x} \leqq \mathfrak{e}_{0}$.

Suppose that for some $s \geqq s_{0}$ and some $p$ we have

$$
W(p, e, s)=w \& p \in \operatorname{range}\left(T_{s}[\mathrm{e}]\right) \& T_{s}^{-1}(p)>\mathrm{e}_{0},
$$

then

$$
W(p, e, s+1)=w \& p \in \operatorname{range}\left(T_{s+1}[e]\right) \& T_{s+1}^{-1}(p)>e_{0} \cdot V \cdot p \in \eta_{s+1} .
$$

Suppose the contrary; then we see at once that $p$ is in the range of $T_{s+1}$ since it is not in $\eta_{s+1}$. If $x$ in the statement of step $s+1$ is not $p$ then $W(p, e, s+1)=W(p, e, s)$ and so either $p$ is not in the range of $T_{s+1}[e]$ or $T_{s+1}^{-1}(p) \ngtr e_{0}$. By choice of $s_{0}$ we have $T_{s+1}^{-1}(p)>\mathfrak{e}_{0}$, therefore $p$ is not in the range of $T_{s+1}[\mathrm{e}]$, which is impossible. Hence $x=p$ in step $s+1$. If $y$ in the statement of step $s+1$ is $>e$ then $W(p, e, s+1)$ $=W(p, e, s)$, also $\mathfrak{x}_{0}$ and $\mathfrak{x}_{1}$ if defined are proper extensions of $\mathrm{e}$. This means that $p$ is in the range of $T_{s+1}[\mathrm{e}]$, because $T_{s+1}$ can only disagree with $T_{s}$ on extensions of $\mathfrak{x}_{0}$. Now $T_{s+1}^{-1}(p)>\mathrm{e}_{0}$ as before, and so $y \leqq e$. But we have $W(p, e, s) \geqq W\left(T\left(\mathrm{e}_{0}\right), e, s\right)$ and so $W\left(T\left(\mathrm{e}_{0}\right), y, s\right)<W(p, y, s+1)$. It follows that $\mathfrak{x}_{1}$ is defined and $\leqq \mathrm{e}_{0}$. Since $T_{s+1}\left(\mathfrak{x}_{1}\right) \neq T_{s}\left(\mathfrak{x}_{1}\right)$ the choice of $s_{0}$ is again contradicted. From this contradiction we infer that (9) does indeed imply (10) when $s \geqq s_{0}$.

It is now apparent that the r.e. set

$$
\left\{u \mid(E z)\left[z \geqq s_{0} \& W(u, e, z)=w \& u \in \operatorname{range}\left(T_{z}[\mathrm{e}]\right) \& T_{z}^{-1}(u)>\mathrm{e}_{0}\right]\right\}
$$


is a subset of $\eta \cup$ range $(T[e])$. It is also clear that all but a finite number of members of $T[e]$ belong to the r.e. set (11). This completes the proof of the lemma.

We can now deduce that $\eta$ is $h h$-simple with no maximal superset. Let $\mathrm{e}$ be a string of length $e$; then from Lemma 7 there exists $w$ such that for all but a finite number of members $x$ of $T[e]$ we have $W(x, e)=w$. If $w$ is even then all but a finite number of members of $T[e]$ are in $\chi_{e}^{\prime}$. If $w$ is odd, then all but a finite number of members of $T[e]$ are in $\chi_{e}^{\prime}$. Let $e_{1}, \ldots, e_{m}$ be all the strings of length $e$ for which the corresponding $w$ 's are even, and let $\mathfrak{e}_{m+1}, \ldots, \mathfrak{e}_{m+n}$ be all the other strings of length $e$ whose corresponding $w$ 's will be odd. Then

$$
\left.\chi_{e} \cap \bigcup \text { range }\left(T\left[\mathrm{e}_{i}\right]\right) \mid 1 \leqq i \leqq m\right\}
$$

and

$$
\left.\bigcup \text { range }\left(T\left[\mathfrak{e}_{i}\right]\right) \mid m<i \leqq m+n\right\}-\chi_{e}
$$

are both finite. Also, the sets

$$
\left.\bigcup \text { range }\left(T\left[\mathrm{e}_{i}\right]\right) \mid 1 \leqq i \leqq m\right\} \quad \bigcup\left\{\operatorname{range}\left(T\left[\mathrm{e}_{i}\right]\right) \mid m<i \leqq m+n\right\}
$$

are disjoint and exhaust $\eta^{\prime}$. Therefore $\eta \cup \chi_{e}^{\prime}$ is r.e. since it differs only finitely from

$$
\bigcup\left\{\eta \cup \operatorname{range}\left(T\left[\mathrm{e}_{i}\right]\right) \mid 1 \leqq i \leqq m\right\} .
$$

Since this is true for any $e$ we have $\eta h h$-simple by Theorem 3. Further, if $\eta \cup \chi_{e}$ is not cofinite then $m>0$ and so $\eta \cup$ range $\left(T\left[e_{1} * 0\right]\right), \eta \cup \operatorname{range}\left(T\left[e_{1} * 1\right]\right)$ are r.e. sets disjoint outside $\eta$ and both intersecting $\left(\eta \cup \chi_{e}\right)^{\prime}$ infinitely. Hence $\eta \cup \chi_{e}$ is not a maximal superset of $\eta$, and so $\eta$ has no maximal superset. We have proved:

THEOREM 5. There exists an hh-simple set with no maximal superset.

Notice that $\mathscr{L}^{*}(\eta)$ is a countable atomless Boolean algebra; this determines its isomorphism type as a Boolean algebra. We shall see in the next section that $\mathscr{L}^{*}(\eta)$ determines $\mathscr{L}(\eta)$, thus there is only one possibility for the lattice of r.e. supersets of $\eta$.

4. The Boolean algebras of supersets of $h h$-simple sets. In this section we show that as $\alpha$ runs through all $h h$-simple sets, so $\mathscr{L}^{*}(\alpha)$ runs through all possible isomorphism types.

Let $\left\{\chi_{i}\right\}$ be any recursive enumeration of all r.e. sets, and let $\alpha$ be a fixed r.e. set. Consider the relation $R$ on the natural numbers defined by

$$
R(x, y) \Leftrightarrow\left(\chi_{x} \cup \alpha\right)-\left(\chi_{y} \cup \alpha\right) \text { is finite. }
$$

Defining $\mathscr{A}=\left\{\alpha_{0}, \alpha_{1}, \ldots\right\}$ where

$$
\alpha_{i}=\{x \mid R(x, i) \& R(i, x)\},
$$

and $\leqq$ on $\mathscr{A}$ by

$$
\alpha_{i} \leqq \alpha_{j} \Leftrightarrow R(i, j),
$$


it is easy to see that the map: $\alpha_{i}$ goes to $\chi_{i} \cup \alpha$, is an isomorphism from $\{\mathscr{A}, \leqq\}$ onto $\left\{\mathscr{L}^{*}(\alpha), \subseteq\right\}$. Turning the argument round, we may define a $\exists \forall \exists$-lattice to be one of the form $\{\mathscr{A}, \leqq\}$ where $\mathscr{A}$ and $\leqq$ are defined from some binary $\exists \forall \exists$-relation $R$ as above, and where there exist binary recursive functions $U, I$ such that for all $x, y$ we have

$$
\alpha_{x} \cup \alpha_{y}=\alpha_{U(x, y)}, \quad \alpha_{x} \cap \alpha_{y}=\alpha_{I(x, y)} .
$$

Since $U, I$ obviously exist above, for any r.e. set $\alpha$ we have $\mathscr{L}^{*}(\alpha)$ isomorphic to a $\exists \forall \exists$-lattice.

It is not generally true that given a $\exists \forall \exists$-lattice which has a 0 (least element) and a 1 (greatest element) we can find an r.e. set $\alpha$ such that $\mathscr{L}^{*}(\alpha)$ is isomorphic to it. For there is a $\exists \forall \exists$-lattice with a 0 and 1 and just one other element, and we have already seen that if $\mathscr{L}^{*}(\alpha)$ is finite then $\alpha \simeq \nu$ or $\mathscr{L}^{*}(\alpha)$ is a Boolean algebra and thus has as order some power of 2 . However, by modifying the construction in the last section we can prove:

THEOREM 6. Let $\mathscr{A}$ be a $\exists \forall \exists$-lattice which is a Boolean algebra, then there is an hh-simple set $\alpha$ such that $\mathscr{L}^{*}(\alpha)$ is isomorphic to $\mathscr{A}$.

Proof. Let $\mathfrak{x}$ be a string of length $x$; we henceforth identify $\mathfrak{x}$ with the finite function defined by:

$$
\begin{aligned}
\mathfrak{x}(n)= & (n+1) \text { th member of string } \mathfrak{x} \text { if } n<x, \\
& \text { undefined otherwise. }
\end{aligned}
$$

Let $\mathscr{A}$ be a countable Boolean algebra. A sequence $\left\{\alpha_{0}, \alpha_{1}, \ldots\right\}$ of members of $\mathscr{A}$ is said to generate $\mathscr{A}$ if every member of $\mathscr{A}$ can be obtained from members of the sequence by the operations of union, intersection, and complementation. A mapping $F$ of $\mathfrak{L}$ into $\{0,1\}$ is said to be an associate of $\mathscr{A}$ if there is a sequence $\left\{\alpha_{0}, \alpha_{1}, \ldots\right\}$ which generates $\mathscr{A}$ such that

$$
F(\mathfrak{x})=1 \Leftrightarrow\left[\bigcap\left\{\alpha_{y} \mid \mathfrak{x}(y)=0\right\} \cap \bigcap\left\{\alpha_{y}^{\prime} \mid \mathfrak{x}(y)=1\right\}=\varnothing\right] .
$$

Note that every member of $\mathscr{A}$ can be expressed in the form

$$
\bigcup\left\{\bigcap\left\{\alpha_{y} \mid \mathfrak{x}(y)=0\right\} \cap \bigcap\left\{\alpha_{y}^{\prime} \mid \mathfrak{x}(y)=1\right\} \mid \mathfrak{x} \in \mathfrak{X}\right\}
$$

where $\mathfrak{X}$ is a finite set of strings. Call this set $\alpha(\mathfrak{X})$. Suppose that every string in $\mathfrak{X}$ has length $\leqq n$ and that $\mathfrak{X}_{0}$ is the set

$$
\{\mathfrak{x} \mid \mathfrak{x} \in \mathfrak{L} \& \mathfrak{x} \text { has length } n \&(E \mathfrak{y})[\mathfrak{y} \in \mathfrak{X} \& \mathfrak{y} \subseteq \mathfrak{x}]\},
$$

it is easy to see that $\alpha(\mathfrak{X})=\alpha\left(\mathfrak{X}_{0}\right)$. Let $\mathfrak{Y}$ be another finite set of strings all of length $\leqq n$ and define $\mathfrak{Y}_{0}$ from $\mathfrak{Y}$ as $\mathfrak{X}_{0}$ was defined from $\mathfrak{X}$. We see that

$$
\alpha(\mathfrak{X}) \subseteq \alpha(\mathfrak{Y}) \Leftrightarrow \alpha\left(\mathfrak{X}_{0}\right) \subseteq \alpha\left(\mathfrak{Y}_{0}\right) \Leftrightarrow(\mathfrak{x})\left[\mathfrak{x} \in \mathfrak{X}_{0}-\mathfrak{Y}_{0} \rightarrow F(\mathfrak{x})=1\right] .
$$

It is now apparent that if $\mathscr{A}^{0}, \mathscr{A}^{1}$ are two countable Boolean algebras with a common associate, then $\mathscr{A}^{0}, \mathscr{A}^{1}$ are isomorphic. For let the common associate be $F$ and $\left\{\alpha_{i}^{0}\right\},\left\{\alpha_{i}^{1}\right\}$ be the corresponding generating sequences of $\mathscr{A}^{0}, \mathscr{A}^{1}$ respectively. 
For each finite set of strings $\mathfrak{X}$ we map $\alpha^{0}(\mathfrak{X})$ into $\alpha^{1}(\mathfrak{X})$. It follows from (12) firstly that this constitutes a good definition and secondly that the mapping is an isomorphism.

Let $\mathscr{A}$ be any $\exists \forall \exists$-Boolean algebra defined as above, and let $\alpha_{0}, \alpha_{1}, \ldots$ be its natural enumeration. From the recursive functions $U, I$ giving effective union and intersection in $\mathscr{A}$ it is clear that we can define recursive functions $V, J$ such that

$$
\alpha_{J(\mathfrak{E})}=\bigcap\left\{\alpha_{y} \mid \mathfrak{x}(y)=0\right\}, \quad \alpha_{V(\mathfrak{E})}=\bigcup\left\{\alpha_{y} \mid \mathfrak{x}(y)=1\right\} .
$$

Now we have the equivalences:

$$
F(\mathfrak{x})=1 \Leftrightarrow \alpha_{J(\mathfrak{E})} \cap \alpha_{V(\mathfrak{E})}^{\prime}=\varnothing \Leftrightarrow \alpha_{J(\mathfrak{E})} \leqq \alpha_{V(\mathfrak{x})} .
$$

Thus given $\mathfrak{x}$ we can effectively find a ternary recursive relation $S_{\mathfrak{x}}$ such that

Define

$$
F(\mathfrak{x})=1 \Leftrightarrow(E x)(y)(E z) S_{\mathfrak{x}}(x, y, z) .
$$

$$
\psi_{\mathfrak{x}, x}=\left\{n \mid(E u)\left[u \leqq x \&(y)\left[y \leqq n \rightarrow(E z) S_{\mathfrak{x}}(u, y, z)\right]\right]\right\} .
$$

Call $\sigma$ a segment of $\nu$ if it satisfies

$$
(x)(y)[x<y \& y \in \sigma . \rightarrow x \in \sigma] .
$$

Now $\psi_{\mathfrak{x}, x}$ is r.e. uniformly in $\mathfrak{x}, x$. Also, for fixed $\mathfrak{x}$ the sequence $\psi_{\mathfrak{x}, 0}, \psi_{\mathfrak{x}, 1}, \ldots$ is an increasing sequence of segments of $\nu$. Further, it is easy to see that

$$
F(\mathfrak{x})=1 \Leftrightarrow(E x)(y)\left[y \geqq x \rightarrow \psi_{\mathfrak{x}, x}=\nu\right] .
$$

The string $\mathfrak{y}$ is a segment of the string $\mathfrak{x}$, written $\mathfrak{y} \subseteq \mathfrak{x}$, if $\mathfrak{x}$ is the same as or a proper extension of $\mathfrak{y}$. We let $L(\mathfrak{x})$ denote the length of $\mathfrak{x}$ and define

$$
\psi_{\mathfrak{x}}=\bigcup\left\{\psi_{\mathfrak{y}, \mathfrak{y}} \mid \mathfrak{y} \text { is a segment of } \mathfrak{x} \& y=L(\mathfrak{x})-L(\mathfrak{y})\right) \text {. }
$$

Now $\psi_{\mathfrak{x}}$ is r.e. uniformly in $\mathfrak{x}$ and is a segment of $\nu$. There are two properties of the sequence $\left\{\psi_{\mathfrak{r}}\right\}$. which we shall need below:

$$
\begin{gathered}
F(\mathfrak{x})=1 \Leftrightarrow\left[\psi_{\mathfrak{y}}=\nu \text { for all but a finite number of extensions } \mathfrak{y} \text { of } \mathfrak{x}\right], \\
\psi_{\mathfrak{x}} \text { finite } \rightarrow\left[\psi_{\mathfrak{y}} \text { is finite for every segment } \mathfrak{y} \text { of } \mathfrak{x}\right] .
\end{gathered}
$$

To prove (13) we note that if $F(\mathfrak{x})=1$ then for some $p$ we have $\psi_{\mathfrak{x}, p}, \psi_{\mathfrak{x}, p+1}, \ldots$ all equal to $\nu$. But if $\mathfrak{y}$ is an extension of $\mathfrak{x}$ such that $L(\mathfrak{x})+p \leqq L(\mathfrak{y})$, then one of $\psi_{\mathfrak{x}, p}$, $\psi_{\mathfrak{x}, p+1}, \ldots$ is a summand of $\psi_{\mathfrak{y}}$ and so $\psi_{\mathfrak{y}}=\nu$. Also, if $F(\mathfrak{x})=0$, then from above $\psi_{\mathfrak{x}, 0}, \psi_{\mathfrak{x}, 1}, \ldots$ are all finite. By the definition of associate $F(\mathfrak{x})=0$ implies $F(\mathfrak{y})=0$ for every segment $\mathfrak{y}$ of $\mathfrak{x}$; therefore $F(\mathfrak{x})=0$ implies that every summand of $\psi_{\mathfrak{x}}$ is finite and hence that $\psi_{\mathfrak{x}}$ is finite. Thus (13) is proved when we observe from the definition of associate that $F(\mathfrak{x})=0$ implies either $F(\mathfrak{x} * 0)=0$ or $F(\mathfrak{x} * 1)=0$, because then $F(\mathfrak{y})=0$ for an infinite number of extensions $\mathfrak{y}$ of $\mathfrak{x}$. Now (14) is clearly true, because we have shown that $F(\mathfrak{x})=0$ implies both $\psi_{\mathfrak{x}}$ finite and $F(\mathfrak{y})=0$ for every segment $\mathfrak{y}$ of $\mathfrak{x}$. 
We now carry out a construction like that of the last section of an r.e. set $\eta$ such that $\mathscr{L}^{*}(\eta)$ has $F$ as an associate. This will clearly be sufficient to establish the theorem. Let $\left\{\chi_{i}\right\}$ be a recursive enumeration of all r.e. sets as before. In this construction at each step the clerk enumerates either a pair in $\nu \times \nu$ or a member of $\mathfrak{L}$ but not both. The set of all pairs of $\nu \times \nu$ which he enumerates is $\left\{(x, y) \mid x \in \chi_{y}\right\}$ and each member of this set is enumerated at an infinite number of steps. Each member $\mathfrak{x}$ of $\mathfrak{L}$ is enumerated at $k$ steps where $k$ is the cardinality of $\psi_{\mathfrak{t}}$.

As before in each step $s$ of the construction we define a number tree $T_{s}$ and we let $\eta_{s}$ consist of those numbers not in the range of $T_{s}$. We define $\omega_{y, s}$ just as before, likewise the auxiliary function $W$. We shall also define in step $s$ the function $C_{s}$ mapping $\mathfrak{L} \times \nu$ into $\nu$. The role of $C_{s}$ in the construction is roughly as follows. Say that $m$ enters $n$-state $x$ at step $s$ if $W(m, n, s) \neq x=W(m, n, s+1)$. For any $x$ of length $n, C_{s}(x, x)$ counts the numbers $m$ which before step $s$ have entered $n$-state $x+1$ through $m$ entering $\omega_{n}$ and which have been "used" in the construction. We require $\mathfrak{L}$ to be arranged in an effective sequence $\mathfrak{L}_{0}, \mathfrak{L}_{1}, \ldots$ The construction of $\eta$ is now specified.

Step 0. Define $T_{0}$ as before. Let $C_{0}(\mathfrak{n}, n)=0$ for each pair $(\mathfrak{n}, n)$.

Step $s+1$. There are two cases according as the clerk enumerates at step $s$ a member of $\nu \times \nu$ or a member of $\mathfrak{L}$.

Case 1. Let $(x, y)$ be the pair which the clerk enumerates at step $s$. If $x$ is not a member of $T_{s}$, or if $\mathfrak{x}=T_{s}^{-1}(x)$ has length $\leqq y$, or if $x$ is in $\omega_{y, s}$, or if $\mathfrak{x} \leqq \mathfrak{x}_{0} * \mathfrak{l}_{z}$ where $\mathfrak{x}_{0}$ is the segment of $\mathfrak{x}$ of length $y$ and where $z=C_{s}\left(\mathfrak{x}_{0}, W(x, y, s)\right)$, then define $T_{s+1}=T_{s}$ and $C_{s+1}=C_{s}$. Otherwise, define

$$
\begin{aligned}
C_{s+1}(\mathfrak{n}, n) & =C_{s}(\mathfrak{n}, n)+1 & & \text { if } \mathfrak{n}=\mathfrak{x}_{0} \text { and } n=W(x, y, s), \\
& =C_{s}(\mathfrak{n}, n) & & \text { otherwise. }
\end{aligned}
$$

Also, let $\mathfrak{x}_{1}$ be the least string if any such that $\mathfrak{x}_{0} \subseteq \mathfrak{x}_{1} \subseteq \mathfrak{x}_{0} * \mathfrak{R}_{z}$, and such that $W\left(x_{1}, y, s\right)<W(x, y, s+1)$ where $x_{1}=T_{s}\left(\mathfrak{x}_{1}\right)$. If there is no such $\mathfrak{x}_{1}$ let $T_{s+1}=T_{s}$ again, but if $\mathfrak{x}_{1}$ is defined we set

$$
\begin{aligned}
T_{s+1}(\mathfrak{a}) & =x & & \text { if } \mathfrak{a}=\mathfrak{x}_{1}, \\
& =T_{s}(\mathfrak{a} * 0) & & \text { if } \mathfrak{a} \text { is one of } \mathfrak{x}, \mathfrak{x} * 0, \mathfrak{x} * 00, \ldots, \\
& =T_{s}(\mathfrak{a}) & & \text { otherwise. }
\end{aligned}
$$

Notice that this definition of $T_{s+1}$ gives $\eta_{s+1}=\eta_{s} \cup\left\{x_{1}\right\}$, and that $T_{s+1}=T_{s}$ gives $\eta_{s+1}=\eta_{s}$.

Case 2. Let $\mathfrak{x}$ be the string which the clerk enumerates at step $s$ then we define

$$
\begin{aligned}
T_{s+1}(\mathfrak{a}) & =T_{s}(\mathfrak{a} * 0) & & \text { if } \mathfrak{a} \text { is one of } \mathfrak{x}, \mathfrak{x} * 0, \mathfrak{x} * 00, \ldots, \\
& =T_{s}(\mathfrak{a}) & & \text { otherwise. }
\end{aligned}
$$

If $x=T_{s}(\mathfrak{x})$ we have $\eta_{s+1}=\eta_{s} \cup\{x\}$. 
The construction which we have described is clearly effective. Given $i$ we can effectively enumerate $\eta_{i}$ and find its cardinality. Further, by inspection of step $s+1$ we see $\eta_{s} \subseteq \eta_{s+1}$. Hence $\left\{\eta_{i}\right\}$ is a strongly r.e. increasing sequence and so has r.e. limit $\eta$.

We now prove Lemmas $9-12$ corresponding to Lemmas 5-8 for the easier construction.

LEMMA 9. $\lim _{x} T_{x}(\mathfrak{x})$ exists if and only if $\psi_{\mathfrak{x}}$ is finite.

Proof. If $\psi_{\mathfrak{x}}$ is infinite then the clerk enumerates $\mathfrak{x}$ at an infinite number of steps. If $\mathfrak{x}$ is enumerated at step $s$ then $T_{s+1}(\mathfrak{x}) \neq T_{s}(\mathfrak{x})$ from Case 2. Hence $\lim _{x} T_{x}(\mathfrak{x})$ does not exist when $\psi_{\mathfrak{r}}$ is infinite.

If $\psi_{\mathfrak{x}}$ is finite, then $\psi_{\mathfrak{y}}$ is finite for every segment $\mathfrak{y}$ of $\mathfrak{x}$. This is immediate from (14). Let $\psi_{\mathfrak{b}}$ be finite, then for induction on $\mathfrak{L}$ we may suppose that $\lim _{x} T_{x}(\mathfrak{y})$ exists for every proper segment $\mathfrak{y}$ of $\mathfrak{b}$. Further, for only finitely many $s$ do we have $T_{s+1}(\mathfrak{b}) \neq T_{s}(\mathfrak{b})$ through Case 2 at step $s+1$. For reductio ad absurdum suppose that $T_{s+1}(\mathfrak{b}) \neq T_{s}(\mathfrak{b})$ for infinitely many $s$. By the induction hypothesis for all sufficiently large such $s$ we have $\mathfrak{b}=\mathfrak{x}$ or $\mathfrak{b}=\mathfrak{x}_{1}$ at step $s+1$. Let $b=L(\mathfrak{b})$ and let $e$ be the greatest number such that there is an infinite set $\sigma$ of values of $s$ for which $T_{s+1}(\mathfrak{b}) \neq T_{s}(\mathfrak{b})$ and $W\left(T_{s}(\mathfrak{b}), b, s+1\right)=e$. Since $W(a, b, c)<2^{b+1}, e$ is well defined. For any sufficiently large $s$ in $\sigma$ we cannot have $\mathfrak{b}=\mathfrak{x}_{1}$ at step $s+1$, because this would mean that $W\left(T_{s+1}(\mathfrak{b}), b, s+1\right)>e$ which would contradict the definition of $e$. Thus for all sufficiently large $s$ in $\sigma$ we have $\mathfrak{b}=\mathfrak{x}$. Hence there is a number $y$ such that for all sufficiently large $s$ in $\sigma$ the clerk enumerates $\left(T_{s}(\mathfrak{b}), y\right)$ at step $s$ and such that $T_{s}(\mathfrak{b})$ is not in $\omega_{y, s}$. The number $y$ depends only on $b, e$ and we have $y<b$. Hence for each sufficiently large $s$ in $\sigma$ we have $C_{s+1}\left(\mathfrak{x}_{0}, 2^{y-b} e\right)=C_{s}\left(\mathfrak{x}_{0}, 2^{y-b} e\right)+1$. Therefore as $s$ increases $C_{s}\left(\mathfrak{x}_{0}, 2^{y-b} e\right)$ takes every value, and so there is an infinite set $\tau$ for each member $s$ of which

$$
C_{s+1}\left(\mathfrak{x}_{0}, 2^{y-b} e\right)=C_{s}\left(\mathfrak{x}_{0}, 2^{y-b} e\right)+1=z+1,
$$

where $\mathfrak{x}_{0} * \mathfrak{L}_{z} \supseteq \mathfrak{b}$. For all sufficiently large $s$ in $\tau$ we have $T_{s+1}(\mathfrak{y})=T_{s}(\mathfrak{y})$ for all segments $\mathfrak{y}$ of $\mathfrak{b}$, whence if $\mathfrak{x}_{1}$ is defined in step $s+1$ then $\mathfrak{b} \subseteq \mathfrak{x}_{1}$. Since $\mathfrak{x}_{0} \subseteq \mathfrak{b} \subseteq \mathfrak{x}_{0} * \mathfrak{L}_{z}$ at each step $s+1$ such that $s$ is in $\tau$, for all sufficiently large $s$ in $\tau$ we have

$$
W\left(T_{s+1}(\mathfrak{b}), y, s+1\right) \geqq W\left(T_{s}(\mathfrak{x}), y, s+1\right)=2^{y-b} e+1 .
$$

Hence for all sufficiently large $s$ in $\tau$ we have $W\left(T_{s+1}(\mathfrak{b}), b, s+1\right) \geqq e+2^{b-y}$. This contradicts the choice of $e$ and so the lemma is proved.

Let $T(\mathfrak{x})$ be equal to $\lim _{x} T_{x}(\mathfrak{x})$ when this is defined and be undefined otherwise, let $W(x, y)$ denote $\lim _{z} W(x, y, z)$.

LEMMA 10. Let e be a string of length e. Let $b=T(\mathfrak{b})$ where $\mathfrak{b}$ is an extension of $\mathfrak{e}$. Let $t$ be the greatest number such that the clerk enumerates $(b, e)$ at step $t$ and such that $b$ is not in $\omega_{e, t}$. Then $T_{t}^{-1}(b)$ is an extension of $\mathrm{e}$ and $W(b, e, t+1)=W(b, e)$. 
Proof. The proof of Lemma 6 will serve.

LEMMA 11. Let e be a string of length $e$, then there exists $w$ such that for all but a finite number of extensions $\mathfrak{x}$ of $\mathrm{e}$ either $T(\mathfrak{x})$ is undefined or $W(T(\mathfrak{x}), e)=w$, and such that $\lim _{x} C_{x}(\mathrm{e}, u)$ exists whenever $u \geqq w$.

Proof. Let $e^{-}$be the string of length $e-1$ of which $e$ is an extension, then we may assume there exists $w^{-}$such that for all but a finite number of extensions $\mathfrak{x}$ of $\mathrm{e}^{-}$we have $T(\mathfrak{x})$ undefined or $W(T(\mathfrak{x}), e-1)=w^{-}$. From the definition of $W$ we see that for all but a finite number of extensions $\mathfrak{x}$ of $\mathfrak{e}$ we have $T(\mathfrak{x})$ undefined or

$$
2 w^{-} \leqq W(T(\mathfrak{x}), e) \leqq 2 w^{-}+1 .
$$

We may suppose that $W(T(\mathfrak{x}), e)=2 w^{-}+1$ for infinitely many extensions $\mathfrak{x}$ of $\mathrm{e}$ such that $T(\mathfrak{x})$ is defined; otherwise there is nothing to prove. Let $\mathfrak{a}$ be any extension of e such that $T(\mathfrak{a})$ is defined, then from Lemma $9 T(\mathfrak{y})$ is defined for every segment $\mathfrak{y}$ of $\mathfrak{a}$. Thus there exists $a$ such that for all $x \geqq a$ and all $\mathfrak{y} \subseteq \mathfrak{a}$ we have $T_{x}(\mathfrak{y})=T(\mathfrak{y})$. There are an infinite number of members $b$ of $T[e]$ for which $W(b, e)=2 w^{-}+1$. Each such $b$ is in $\chi_{e}$ and thus has associated with it a greatest $t$ such that $b$ is not in $\omega_{e, t}$ and such that $(b, e)$ is enumerated at step $t$. Since there are infinitely many such $b$ we can find one with $T^{-1}(b)>$ any given member of $\mathfrak{R}$. Since $T_{t}^{-1}(b) \geqq T^{-1}(b)$ for all $t$, and since $e \subseteq T_{t}^{-1}(b)$ by the last lemma, and since $W(b, e, t+1)=2 w^{-}+1$, it follows that $C_{s}\left(e, 2 w^{-}\right)$takes all values as $s$ increases. Thus there exist infinitely many steps $s+1$ such that $C_{s+1}\left(e, 2 w^{-}\right)=C_{s}\left(e, 2 w^{-}\right)+1=z+1$ where $\mathfrak{e} \subseteq \mathfrak{a} \subseteq \mathfrak{e} * \mathfrak{L}_{z}$. Choose any such $s \geqq a$, then since $\mathfrak{x}_{1}$ in step $s+1$ is not a segment of $\mathfrak{a}$ we have

$$
W(T(\mathfrak{a}), e, s) \geqq W(x, e, s+1)=2 w^{-}+1 .
$$

Hence for any extension $\mathfrak{a}$ of $\mathrm{e}$ such that $T(\mathfrak{a})$ is defined we have $W(T(\mathfrak{a}), e)$ $\geqq 2 w^{-}+1$. Since for all but a finite number of extensions $\mathfrak{x}$ of $e$ either $T(\mathfrak{x})$ is undefined or $W(T(\mathfrak{x}), e) \leqq 2 w^{-}+1$, the first part of the lemma is proved. Also, the latter part of the argument shows that, if $\lim _{x} C_{x}(e, u)=\infty$, then $W(T(\mathfrak{a}), e)$ $\geqq u+1$ for any extension $\mathfrak{a}$ of $e$ such that $T(\mathfrak{a})$ is defined. Thus either there are only finitely many extensions $\mathfrak{a}$ of e such that $T(\mathfrak{a})$ is defined in which case $w$ is arbitrary and may certainly be chosen to satisfy the second part of the theorem, or $w$ is fixed by the first part of the theorem and $\lim _{x} C_{x}(\mathrm{e}, u)$ exists for every $u \geqq w$.

LemMa 12. For any string e the set $\eta \cup$ range $(T[e])$ is r.e.

Proof. Let e have length $e$. From Lemma 11 there exist $w, \mathfrak{e}_{0}$ such that $\mathrm{e}_{0}$ is an extension of $e$ and such that for all extensions $\mathfrak{x}$ of $e$ which are $\geqq e_{0}$ we have $T(\mathfrak{x})$ undefined or $W(T(\mathfrak{x}), e)=w$. Let $s_{0}$ be such that for all $\mathfrak{x} \leqq \mathfrak{e}_{0}$ we have $T(\mathfrak{x})$ undefined or $T_{s}(\mathfrak{x})=T(\mathfrak{x})$ for all $s \geqq s_{0}$. Let $\pi$ consist of the set of pairs $(p, s)$ with $s \geqq s_{0}$ which satisfy

$$
W(p, e, s)=w \& p \in \operatorname{range}\left(T_{s}[e]\right) \& T_{s}^{-1}(p)>\mathfrak{e}_{0},
$$


but for which

$$
W(p, e, s+1)=w \& p \in \operatorname{range}\left(T_{s+1}[e]\right) \& T_{s+1}^{-1}(p)>\mathfrak{e}_{0} \cdot \vee \cdot p \in \eta
$$

is false. Suppose $(p, s)$ is in $\pi$. If Case 2 occurs at step $s+1$, then $T_{s}^{-1}(p)$ is one of $x$, $\mathfrak{x} * 0, \mathfrak{x} * 00, \ldots$, where $\mathfrak{x}$ is the member of $\mathfrak{L}$ enumerated by the clerk at step $s$. Since (16) fails, we have $p \notin \eta$ and so $T_{s}^{-1}(p) \neq \mathfrak{x}$. From (15), $T_{s}^{-1}(p) \supseteq e$ and $T_{s}^{-1}(p)>\mathrm{e}_{0} \supseteq \mathrm{e}$. Hence $T_{s}^{-1}(p)$ is a proper extension of $e$ and so $p$ is in the range of $T_{s+1}[e]$. Also, $W(p, e, s+1)=W(p, e, s)$ whence for (16) to fail we must have $T_{s+1}^{-1}(p) \leqq e_{0}$. But by choice of $s_{0}$ we know that $T\left(T_{s+1}^{-1}(p)\right)$ is not defined, and we can prove by induction that $T\left(T_{t}^{-1}(p)\right)$ is not defined for any $t>s$. This is a contradiction and so Case 1 obtains at step $s+1$. We can see again that $p \notin \eta$ and hence that $T_{s}^{-1}(p)$ is one of $\mathfrak{x}, \mathfrak{x} * 0, \mathfrak{x} * 00, \ldots$ Now $T_{s+1}^{-1}(p)>\mathfrak{e}_{0}$ for the same reason as before; it follows that $T_{s}^{-1}(p)$ cannot be any of $\mathfrak{x} * 0, \mathfrak{x} * 00, \ldots$, because (16) fails. Thus $T_{s}^{-1}(p)=\mathfrak{x}$ and either $W(p, e, s+1) \neq w$ or $T_{s+1}^{-1}(p)=\mathfrak{x}_{1} \notin T_{s+1}[\mathrm{e}]$. We conclude that at step $s$ the clerk enumerates a pair $(p, y)$ with $y \leqq e$.

We may regard $\pi$ as a function of $\left(e_{0}, s_{0}\right)$ where this pair ranges through all those satisfying the conditions imposed on $\left(e_{0}, s_{0}\right)$ above. If $\pi$ contains $(p, s)$ such that the clerk enumerates $(p, y)$ at step $s$, then we say that $\left(e_{0}, s_{0}\right)$ corresponds to $y$. We say that $e_{0}$ corresponds to $y$ if $\left(\mathrm{e}_{0}, s_{0}\right)$ corresponds to $y$ for infinitely many values of $s_{0}$. Since $y \leqq e$, either $\pi$ is empty for some pair $\left(e_{0}, s_{0}\right)$, or there exists $y$ to which infinitely many values of $e_{0}$ correspond. Suppose the latter. Let $e_{1}$ be the segment of $e$ of length $y$, and let $w_{1}$ be the value of $W(y, p, s)$ corresponding to $W(p, e, s)=w$. If $C_{s}\left(e_{1}, w_{1}\right)$ is bounded as $s$ increases, let $\lim _{x} C_{x}\left(e_{1}, w_{1}\right)=z$ and suppose that $C_{s}\left(e_{1}, w_{1}\right)=z$ for all $s \geqq s_{1}$. Since infinitely many $e_{0}$ correspond to $y$ we can find $\left(e_{0}, s_{0}\right)$ corresponding to $y$ such that $e_{0} \geqq e_{1} * \mathfrak{L}_{z}$, such that $L\left(e_{0}\right)>y$, and such that $s_{0} \geqq s_{1}$. Now take $(p, s)$ in $\pi$ such that the clerk enumerates $(p, y)$ at step $s$. Clearly, we have $C_{s+1}\left(e_{1}, w_{1}\right)=C_{s}\left(e_{1}, w_{1}\right)+1$ which contradicts $s \geqq s_{0} \geqq s_{1}$. Hence $\lim _{x}$ $C_{x}\left(e_{1}, w_{1}\right)=\infty$. It now follows from the last lemma that for all but a finite number of extensions $\mathfrak{x}$ of $\mathfrak{e}_{1}$ we have $T(\mathfrak{x})$ undefined or $W(T(\mathfrak{x}), y)>w_{1}$. But $W(T(\mathfrak{x}), y)>w_{1}$ implies $W(T(\mathfrak{x}), e)>w$; and so from the last lemma $T(\mathfrak{x})$ is defined for only a finite number of extensions $\mathfrak{x}$ of $\mathrm{e}$. If this case the conclusion of the theorem is immediate.

There remains the case in which $\pi$ is empty for some pair $\left(e_{0}, s_{0}\right)$. Fix on any such pair, then since (15) implies (16) for $s \geqq s_{0}$, the r.e. set

$$
\left\{u \mid(E z)\left[z \geqq s_{0} \& W(e, u, z)=w \& u \in \operatorname{range}\left(T_{z}[\mathrm{e}]\right) \& T_{z}^{-1}(u)>e_{0}\right]\right\}
$$

is a subset of $\eta \cup$ range $(T[e])$. However, it is also clear that all but a finite number of members of $T[e]$ belong to the r.e. set (17). This completes the proof of the lemma.

We can repeat the argument following the proof of Lemma 8 to prove $\eta \cup \chi_{e}^{\prime}$ is r.e., and that there are strings $e_{m+1}, \ldots, e_{m+n}$ of length $e$, where $n$ may be zero, such that

$$
\eta \cup \chi_{e} \simeq \eta \cup \cup\left\{\operatorname{range}\left(T\left[\mathrm{e}_{i}\right]\right) \mid m<i \leqq m+n\right\}
$$


Let $\eta \cup$ range $(T[\mathrm{e}])$ be denoted by $\rho(\mathrm{e})$. We have now shown that the equivalence classes of the form $\{\rho(e)\}$ generate the whole of $\mathscr{L}^{*}(\eta)$. For $i \geqq 0$, let

$$
\rho_{i}=\bigcup\{\rho(\mathfrak{x} * 0) \mid L(\mathfrak{x})=i\} \text {. }
$$

Let $e$ be a string of length $e$, and define $\sigma_{i}$ for $i<e$ by

$$
\begin{aligned}
\sigma_{i} & =\rho_{i} \quad \text { if } \mathrm{e}(i)=0, \\
& =\rho_{i}^{\prime} \quad \text { if } \mathrm{e}(i)=1 .
\end{aligned}
$$

It is clear that $\rho(e)=\bigcap\left\{\sigma_{i} \mid i<e\right\}$. Thus the sequence $\left\{\rho_{0}\right\},\left\{\rho_{1}\right\}, \ldots$, generates $\mathscr{L}^{*}(\eta)$. Let $G$ be the corresponding associate of $\mathscr{L}^{*}(\eta)$, then we have

$$
G(e)=1 \Leftrightarrow\left[\bigcap\left\{\left\{\rho_{y}\right\} \mid e(y)=0\right\} \cap \bigcap\left\{\left\{\rho_{y}^{\prime}\right\} \mid e(y)=1\right\}=\{\eta\}\right] .
$$

The condition on the right is the same as $\rho(e) \simeq \eta$, which is the same as: $T(\mathfrak{x})$ is defined for only finitely many extensions $x$ of $e$. From Lemma 9 this is the same as: $\psi_{\varepsilon}$ is finite for only finitely many extensions $x$ of e. From (13) this is the same as: $F(e)=1$. Thus $\mathscr{L}^{*}(\eta)$ and $\mathscr{A}$ are isomorphic since they have a common associate. This completes the proof of the theorem.

D. A. Martin has shown that the degrees of $h h$-simple sets are just those r.e. degrees $\boldsymbol{a}$ which satisfy $\boldsymbol{a}^{\prime}=\mathbf{0}^{\prime \prime}$, and also that any of these degrees is the degree of some maximal set. His theorem can be combined with that just proved to yield: given any r.e. degree $\boldsymbol{a}$ such that $\boldsymbol{a}^{\prime}=\boldsymbol{0}^{\prime \prime}$ and any $\exists \forall \exists$-Boolean algebra $\mathscr{A}$ we can find an $h h$-simple set $\eta$ of degree $a$ such that $\mathscr{A} \cong \mathscr{L}^{*}(\eta)$. Further, $\eta$ can also be chosen so that all coinfinite supersets of $\eta$ have degree $a$. Recently R. W. Robinson has proved that if $\boldsymbol{a}^{\prime}=\mathbf{0}^{\prime}$ then any r.e. set of degree $\boldsymbol{a}$ has a maximal superset. For "maximal superset" we can read " $h$-simple set $\eta$ with $\mathscr{L}^{*}(\eta) \cong \mathscr{A}$ ".

One interesting consequence of Theorem 6 is the existence of a set of natural numbers $\zeta$ such that the lattice of sets r.e. in $\zeta$ is not isomorphic to the lattice of r.e. sets. With each ordinal $n$ we associate a Boolean algebra $\mathscr{L}^{n}$ as follows: $\mathscr{L}^{n}$ is to have all the ordinals $<n$ as generators, and its inclusion is defined by the relations $n_{1} \subseteq n_{2}$ for all pairs of ordinals $\left(n_{1}, n_{2}\right)$ such that $n_{1} \leqq n_{2}$. An ordinal $p$ is said to be imbeddable in a lattice $\mathscr{L}$ if there is a (1-1) strictly monotonic map from the set of ordinals $<p$ into $\mathscr{L}$.

Consider the Boolean algebra $\mathscr{L}^{n}$ as a lattice. Every element of the lattice can be expressed uniquely in one of the five forms:

$$
\begin{aligned}
& \varnothing, \\
& \bigcup\left\{n_{2 i+2}-n_{2 i+1} \mid i=0,1, \ldots, j-1\right\}, n_{0} \cup \cup\left\{n_{2 i+2}-n_{2 i+1} \mid i=0,1, \ldots, j-1\right\}, \\
& \bigcup\left\{n_{2 i+2}-n_{2 i+1} \mid i=0,1, \ldots, j-1\right\} \cup\left(n_{2 j+1}^{\prime}\right), \\
& n_{0} \cup \bigcup\left\{n_{2 i+2}-n_{2 i+1} \mid i=0,1, \ldots, j-1\right\} \cup\left(n_{2 j+1}^{\prime}\right), \\
& 1,
\end{aligned}
$$

where $j$ is a natural number, $n_{0}, n_{1}, \ldots, n_{2 j+1}$ is a strictly increasing sequence of ordinals $<n$, and 1 is the universe. We say that an element $\alpha$ of $\mathscr{L}^{n}$ contains the ordinal $m<n$ if $\alpha$ is of the second or third forms with $m \leqq n_{2 j}$, or is of the fourth or fifth forms. 
LEMMA 13. If $m$ is an ordinal imbeddable in $\mathscr{L}^{n}$ then $m \leqq 2^{n+1}$.

Proof. We prove this by induction on $n$. For $n$ finite we leave it to the reader. Let $k$ be an infinite ordinal; then $\mathscr{L}^{k}$ is isomorphic to $\mathscr{L}^{k+1}$. For we can map the generator $p$ of $\mathscr{L}^{k+1}$ into $(p+1)-0$ in $\mathscr{L}^{k}$ if $p$ is finite, $p$ in $\mathscr{L}^{k+1}$ into $p-0$ in $\mathscr{L}^{k}$ if $p$ is infinite and $<k$, and $k$ into $0^{\prime}$. Thus, if the lemma holds for $n=k$, it holds for $n=k+1$. Now suppose that $k$ is a limit ordinal, and that the lemma holds for all $n<k$. Let $\alpha_{0}, \alpha_{1}, \ldots$, be a strictly increasing sequence in $\mathscr{L}^{k}$ of order type $m$. Let $q$ be the least ordinal if any such that $\alpha_{q}$ complements a generator of $\mathscr{L}^{k}$. If $q$ is undefined then any proper segment of $\alpha_{0}, \alpha_{1}, \ldots$, omits or has last member $\alpha_{s}$ for some $s<m$ and so can be imbedded in $\mathscr{L}^{t}$ where $t$ is the greatest ordinal contained in $\alpha_{s}$. Thus in this case from the induction hypothesis $m \leqq$ $\sup \left\{2^{n+1} \mid n<k\right\} \leqq 2^{k}$. If $q$ is defined then either $\alpha_{q}$ is 1 or $\alpha_{q}$ is a member of $\mathscr{L}^{k}$ expressible in either the second or third form. If $\alpha_{q}=1$ then $q+1=m$ and deleting $\alpha_{q}$ from the sequence gives one for which $q$ is not defined. Thus by the previous argument $q \leqq 2^{k}$, and so $m \leqq 2^{k+1}$. Suppose finally that $\alpha_{q} \neq 1$ and that it complements the generator $r$ of $\mathscr{L}^{k}$. Consider the sequences $\left\{\alpha_{x} \mid x<q\right\}$ and $\left\{\alpha_{x} \mid x \geqq q\right\}$ separately. The first has order type $\leqq 2^{k}$ from the same reasoning as before, and the second can be imbedded in $\mathscr{L}^{r}$ and so has order type $\leqq 2^{k}$ by the induction hypothesis. Thus the order type of the whole sequence is again $\leqq 2^{k+1}$. This completes the proof of the lemma.

Although it is a matter of no importance for the present paper, we are curious to know whether $m \leqq 2^{n+1}$ can be replaced by $m<2 n$ as the conclusion of this lemma for $n$ infinite.

Consider the Boolean algebras $\mathscr{L}^{*}(\alpha)$ with $\alpha h h$-simple. Let $P$ be the set of countable ordinals $m$ such that there is an $h h$-simple set $\alpha$ with $\mathscr{L}^{*}(\alpha)$ isomorphic to $\mathscr{L}^{m}$. From the last lemma it follows that a fixed $h h$-simple set $\alpha$ can only give rise to a countable number of members of $P$. Hence $P$ is countable and there is a least ordinal $p$ not in $P$. For no $h h$-simple set $\alpha$ is $\mathscr{L}^{*}(\alpha)$ isomorphic to $\mathscr{L}^{p}$, but since $p$ is countable there is a well-ordering of the natural numbers of order type $p$. The order relation of this ordering is recursive in some set $\zeta$ and $\mathscr{L}^{p}$ is a $\exists \forall \exists$-algebra relative to $\zeta$, i.e., when we replace the notion of recursiveness by that of recursiveness in $\zeta$. Thus for some set $\alpha$ r.e. in $\zeta$ we have $\mathscr{L}^{*}(\alpha) \cong \mathscr{L}^{p}$ and so the lattice of sets r.e. in $\zeta$ is not isomorphic to the lattice of r.e. sets.

By characterising the lattices $\mathscr{L}^{*}(\alpha)$ with $\alpha h$-simple we have characterised the lattices $\mathscr{L}(\alpha)$; this follows from:

Lemma 14. Let $\mathscr{L}_{1}, \mathscr{L}_{2}$ be countable sublattices of $\mathscr{N}$ such that $\mathscr{L}_{1}^{*}, \mathscr{L}_{2}^{*}$ are isomorphic sublattices of $\mathscr{N}^{*}$. If

$$
\left[\varnothing \in \mathscr{L}_{1} \Leftrightarrow \varnothing \in \mathscr{L}_{2}\right] \&\left[\nu \in \mathscr{L}_{1} \Leftrightarrow \nu \in \mathscr{L}_{2}\right]
$$

then $\mathscr{L}_{1} \cong \mathscr{L}_{2}$. 
Proof. If $\alpha$ is a member of $\mathscr{N}$ let $\alpha^{*}$ denote the corresponding member of $\mathscr{N}^{*}$. Let $\Phi$ be an isomorphism from $\mathscr{L}_{1}^{*}$ onto $\mathscr{L}_{2}^{*}$. Choose any enumerations of $\mathscr{L}_{1}$, $\mathscr{L}_{2}$ and suppose first of all that neither $\varnothing$ nor $\nu$ is in $\mathscr{L}_{1}$ or $\mathscr{L}_{2}$. We construct sequences $\left\{\beta_{i}^{1}\right\},\left\{\beta_{i}^{2}\right\}$ enumerating $\mathscr{L}_{1}, \mathscr{L}_{2}$ respectively. Suppose that $\beta_{i}^{1}, \beta_{i}^{2}$ have already been defined for all $i<k$ and that $m$ steps of the construction have been performed. Suppose further that $\left\{\beta_{i}^{1} \mid i<k\right\},\left\{\beta_{i}^{2} \mid i<k\right\}$ are sublattices of $\mathscr{L}_{1}, \mathscr{L}_{2}$ respectively, and that for all $i<k$ and $j<k$ we have $\Phi\left(\beta_{i}^{1 *}\right)=\beta_{i}^{2 *}$ and

$$
\text { cardinality }\left(\beta_{i}^{1}-\beta_{j}^{1}\right)=\text { cardinality }\left(\beta_{i}^{2}-\beta_{j}^{2}\right) \text {. }
$$

If $m$ is even, let $\beta_{k}^{1}$ be the first member of $\mathscr{L}_{1}$ which is not in $\left\{\beta_{i}^{1} \mid i<k\right\}$. Since $\Phi$ is an isomorphism we have

$$
\begin{aligned}
& \bigcap\left\{\beta_{i}^{1} \mid i<k\right\}-\beta_{k}^{1} \text { finite } \Leftrightarrow \Phi\left(\beta_{k}^{1 *}\right) \supseteq \bigcap\left\{\beta_{i}^{2 *} \mid i<k\right\}, \\
& \beta_{k}^{1}-\bigcup\left\{\beta_{i}^{1} \mid i<k\right\} \text { finite } \Leftrightarrow \bigcup\left\{\beta_{i}^{2 *} \mid i<k\right\} \supseteq \Phi\left(\beta_{k}^{1 *}\right),
\end{aligned}
$$

and for $i, j$ both $<k$

$$
\begin{gathered}
\beta_{k}^{1} \cap\left(\beta_{i}^{1}-\beta_{j}^{1}\right) \text { finite } \Leftrightarrow \Phi\left(\beta_{k}^{1 *}\right) \cap \beta_{i}^{2 *} \subseteq \beta_{j}^{2 *}, \\
\left(\beta_{i}^{1}-\beta_{j}^{1}\right)-\beta_{k}^{1} \text { finite } \Leftrightarrow \beta_{i}^{2 *} \subseteq \Phi\left(\beta_{k}^{1 *}\right) \cup \beta_{j}^{2 *} .
\end{gathered}
$$

Considering $\nu$ as the union of $\left(\bigcup\left\{\beta_{i}^{1} \mid i<k\right\}\right)^{\prime}, \bigcap\left\{\beta_{i}^{1} \mid i<k\right\}$, and the minimal nonempty sets of the form $\beta_{i}^{1}-\beta_{j}^{1}$ with $i, j$ both $<k$, we see that there exists a representative $\beta_{k}^{2}$ of $\Phi\left(\beta_{k}^{1 *}\right)$ such that (21) is satisfied for all $i, j$ both $\leqq k$. Now let $\beta_{k+1}^{1}, \ldots, \beta_{p}^{1}$ be the remaining members of $\mathscr{L}_{1}$ obtainable from $\beta_{0}^{1}, \ldots, \beta_{k}^{1}$ by taking unions and intersections, and let $\beta_{k+1}^{2}, \ldots, \beta_{p}^{2}$ be respectively the corresponding members of $\mathscr{L}_{2}$. This completes step $m$, and the induction hypothesis for step $m+1$ will be satisfied. If $m$ is odd we interchange the superscripts 1,2 and the subscripts 1,2 which appear above. It is clear from (18) that mapping $\beta_{i}^{1}$ into $\beta_{i}^{2}$ for all $i$ gives an isomorphism from $\mathscr{L}_{1}$ onto $\mathscr{L}_{2}$.

If $\varnothing$ is in both of $\mathscr{L}_{1}, \mathscr{L}_{2}$ we let $\beta_{0}^{1}=\beta_{0}^{2}=\varnothing$; if $\nu$ is in both of $\mathscr{L}_{1}, \mathscr{L}_{2}$ we let $\beta_{0}^{1}=\beta_{0}^{2}=\nu$, unless $\varnothing$ is also present in which case let $\beta_{1}^{1}=\beta_{1}^{2}=\nu$. Otherwise we proceed as before. This completes the proof of the lemma.

To see from this lemma that $\mathscr{L}^{*}(\alpha)$ fixes $\mathscr{L}(\alpha)$ for $\alpha h h$-simple, we simply observe that there is an isomorphism between $\mathscr{N}$ and the lattice of all supersets of $\alpha$.

5. Some results concerning non- $h h$-simple r.e. sets. In this section we give some examples to show that the method for constructing $h h$-simple sets developed above, which may be regarded as an extension of Friedberg's maximal set construction, is also fruitful in certain other contexts.

Let $\alpha$ be an r.e. set, an r.e. subset $\beta$ of $\alpha$ is called a major subset of $\alpha$ if $\alpha-\beta$ is infinite and if for any r.e. set $\gamma \alpha \cup \gamma=\nu$ implies $\beta \cup \gamma \simeq \nu$.

THEOREM 7. Every nonrecursive r.e. set has a major subset.

Proof. Let $\alpha$ be any nonrecursive r.e. set, and let $\left\{\alpha_{i}\right\}$ be a strongly r.e. increasing sequence of finite sets whose limit is $\alpha$. Let a clerk simultaneously enumerate all 
r.e. sets in the sequence $\left\{\chi_{i}\right\}$ as in $\S 3$ above, and let $\left\{\chi_{i, s}\right\}$ be the set of numbers enumerated in $\left\{\chi_{i}\right\}$ by the end of step s. Define $F$ by

$$
F(i, s)=\min \alpha_{s}^{\prime} \cap \chi_{i, s}^{\prime} .
$$

Notice that $F(i, s)$ is increasing in $s$ for fixed $i$, and that $\lim _{x} F(i, x)=\infty$ if and only if $\alpha \cup \chi_{i}=\nu$.

We enumerate a major subset $\beta$ of $\alpha$ as the clerk enumerates the sets $\chi_{i}$; let $\beta_{s}$ be the finite set of numbers which have been enumerated in $\beta$ by the end of step $s$. Define $W$ by

$$
W(x, y, s)=\sum\left\{2^{y-z} \mid x \in \chi_{z, s} \& z \leqq y \& x<F(z, s)\right\} .
$$

Define the protected sequence $B(0, s), B(1, s), \ldots$ of $\alpha_{s}-\beta_{s}$ by

$$
B(0, s)=\min \left\{x \mid x \in \alpha_{s}-\beta_{s} \& W(x, 0, s)=\max \left\{W(y, 0, s) \mid y \in \alpha_{s}-\beta_{s}\right\}\right\}
$$

and for $i>0$

$$
\begin{aligned}
B(i, s)= & \min \left\{x \mid x \in \alpha_{s}-\beta_{s} \& x>B(i-1, s)\right. \\
& \left.\& W(x, i, s)=\max \left\{W(y, i, s) \mid y \in \alpha_{s}-\beta_{s} \& y>B(i-1, s)\right\}\right\} .
\end{aligned}
$$

Because $\alpha_{s}-\beta_{s}$ is finite, $B(i, s)$ will only be defined for finitely many $i$ for a given $s$. The construction is now very simple. At step 0 we do nothing, at step $s+1$ we enumerate in $\beta$ all the members of $\alpha_{s}-\beta_{s}$ which are not in its protected sequence.

LEMMA 15. Let $i$ be fixed then $B(i, s)$ is defined for all sufficiently large $s$ and $\lim _{x} B(i, x)$ exists.

Proof. By induction on $i$. Suppose the lemma is true for all $i<e$, then there exist $f$ and numbers $b_{0}, b_{1}, \ldots, b_{e-1}$ such that for all $s \geqq f$ and all $i<e$ we have $B(i, s)$ defined and equal to $b_{i}$. If $s>f$ and $\alpha_{s}-\beta_{s}$ contains a number $>b_{e-1}$ then $B(e, s)$ is defined by inspection of (20). (The adjustments for $e=0$ are left to the reader.) Such steps $s$ exist, because $\alpha$ is infinite and because $\beta_{s} \subseteq \alpha_{s-1}$ for all $s>0$. Further, if $B(e, s)$ is defined for some $s>f$ then $\alpha_{s+1}-\beta_{s+1}$ contains a number $>b_{e-1}$, namely $B(e, s)$, and so $B(e, s+1)$ is also defined. Thus $B(e, s)$ is defined for all sufficiently large $s$.

If $B(e, s+1), B(e, s)$ are both defined and different for some $s>f$ then from (20)

$$
\begin{aligned}
W(B(e, s+1), e, s+1) & >W(B(e, s), e, s+1) \cdot \vee \cdot W(B(e, s+1), e, s+1) \\
& =W(B(e, s), e, s+1) \& B(e, s+1)<B(e, s) .
\end{aligned}
$$

Because $W(x, y, s)$ is increasing in $s$, we can replace $W(B(e, s), e, s+1)$ by $W(B(e, s), e, s)$. Thus $W(B(e, s), e, s)$ increases with $s$, and since $W(x, y, s)<2^{y+1}$, $\lim _{x} W(B(e, x), e, x)$ exists. Once this limit is attained $B(e, s+1) \neq B(e, s)$ only when $B(e, s+1)<B(e, s)$. Therefore $\lim _{x} B(e, x)$ exists and the lemma is proved.

The lemma shows that $\alpha-\beta$ is infinite. We complete the proof of the theorem by showing that any r.e. complement of $\alpha$ is almost a complement of $\beta$. For reductio 
ad absurdum let $\chi_{e}$ be the first of $\chi_{0}, \chi_{1}, \ldots$ such that $\alpha \cup \chi_{e}=\nu$ and $\beta \cup \chi_{e}$ is coinfinite. Let $\gamma=\left\{x \mid x<e \& \alpha \cup \chi_{x}=\nu\right\}$. Let $W(x, y) \operatorname{denote}_{\lim _{z}} W(x, y, z)$. Let $b_{0}, b_{1}, \ldots$ be the enumeration of $\alpha-\beta$ in order of magnitude. Letting $s$ tend to $\infty$ in (20) we have

$$
(x)\left[x \geqq i \rightarrow W\left(b_{x}, i\right) \leqq W\left(b_{i}, i\right)\right] .
$$

Also, from (19) for any numbers $m, n, i$ we have

$$
(x)[x \leqq i \rightarrow[W(m, i) \geqq W(n, i) \rightarrow W(m, x) \geqq W(n, x)]] .
$$

Thus it follows that

$$
W\left(b_{i}, i\right) \geqq W\left(b_{i+1}, i\right) \geqq W\left(b_{i+2}, i\right) \geqq \cdots
$$

and so $\lim _{x} W\left(b_{x}, i\right)$ exists for all $i$. If $x$ is in $\gamma$ then $\alpha-\left(\beta \cup \chi_{x}\right)$ is finite and so for all but a finite number of $b$ in $\alpha-\beta$

$$
b \in \chi_{x} \& x \leqq e \& b \leqq \lim _{y} F(x, y) .
$$

If $x<e$ and $x$ is not in $\gamma$ then only a finite number of values of $b$ satisfy (21), because $\lim _{y} F(x, y)$ exists. If $x=e$ then $\alpha-\left(\beta \cup \chi_{x}\right)$ is infinite and so there are an infinite number of members $b$ of $\alpha-\beta$ which do not satisfy (21). Hence by comparing (19) and (21) we see that

$$
W(b, e)=\sum\left\{2^{e-x} \mid x \in \gamma\right\}
$$

for infinitely many $b$ in $\alpha-\beta$. Since for all but a finite number of values of $b$ in $\alpha-\beta$ the value $W(b, e)$ is the same we see that (22) holds for all but finitely many $b$ in $\alpha-\beta$. Let $\lambda$ be the r.e. set consisting of those numbers which are enumerated in $\bigcap\left\{\chi_{x} \mid x \in \gamma \vee x=e\right\}$ before they are enumerated in $\beta$, that is

$$
\lambda=\left\{x \mid(E y)(z)\left[z \in \gamma \vee z=e . \rightarrow . x \in \chi_{z, y} \& x \notin \beta_{y}\right]\right\} .
$$

Clearly $\lambda \supseteq \alpha^{\prime}$, and so $\lambda \cap \alpha$ must be infinite since $\alpha$ is not recursive. Let $b_{f}$ be the least member of $\alpha-\beta$ with $f>e$ such that $b=b_{f}$ satisfies (22), and let $g$ be such that $B(x, s)=b_{x}$ for all $x \leqq f$ and $s \geqq g$. Since $\lambda \cap \alpha$ is infinite, there exist $m, s$ such that $m>b_{f}, s>g, m<F(z, s)$ if $z \in \gamma$ or $z=e$, and

$$
m \in\left(\alpha_{s}-\beta_{s}\right) \cap \bigcap\left\{\chi_{z, s} \mid z \in \gamma \vee z=e\right\} .
$$

This implies that

$$
W(m, e, s)=\sum\left\{2^{e-z} \mid z \in \gamma \vee z=e\right\}>\sum\left\{2^{e-z} \mid z \in \gamma\right\}=W\left(b_{f}, e\right) .
$$

Hence $W(m, f, s)>W\left(b_{f}, f\right)$ and so by the definition of $B(f, s)$ we have $B(f, s)>b_{f}$, which contradicts the definition of $b_{f}$. This contradiction completes the proof of the theorem.

We can easily obtain from this theorem a coinfinite r.e. set $\beta$ which has no $h h$ simple superset. (Martin showed in [4] that we can find such $\beta$ including any given 
nonhypersimple set, and further $\beta$ can be made nonhypersimple or hypersimple. These stronger results we do not obtain.) Let $\alpha$ be any nonrecursive set and $\beta$ be any major subset of $\alpha$. Let $F$ be a (1-1) recursive function mapping $\nu$ onto $\alpha$, then $F^{-1}(\beta)$ has no $h h$-simple superset. To prove this it is sufficient to show that neither $\beta$ nor any r.e. set between $\beta$ and $\alpha$ is $h h$-simple in $\alpha$. It follows from Theorem 2 that either $\beta$ is not $h h$-simple in $\alpha$ or there exists a recursive set $\delta$ such that $\beta \cup \delta=\alpha$. In the latter case $\delta^{\prime}$ is an r.e. complement of $\alpha$ such that $\delta^{\prime} \cup \beta$ is coinfinite, and so we have $\beta$ non- $h h$-simple in $\alpha$. If $\gamma$ is any r.e. set satisfying $\beta \subseteq \gamma \subseteq \alpha$ and $\alpha-\gamma$ infinite, then $\gamma$ is a major subset of $\alpha$ and so is also non- $h h$-simple in $\alpha$.

There is one minor question left open in Martin's paper which can be resolved very easily, using the last theorem. Given a non- $h h$-simple set $\beta$ it is not always possible to find a coinfinite r.e. superset $\alpha$ of $\beta$ such that $\alpha$ has no $h h$-simple superset. For let $\alpha$ be a maximal r.e. set and $\beta$ be a major subset of it, then $\beta$ is not $h h$-simple because it is not $h h$-simple in $\alpha$. Further, if $\gamma$ is any r.e. superset of $\beta$, then either $\gamma$ is confinite or $\gamma-\alpha$ is finite. Thus there is no coinfinite r.e. superset of $\beta$ which has no $h$ h-simple superset.

For the next theorem we need a result of Martin [5, p. 273] which states that if there is a recursive function $F$ and an r.e. set $\sigma$ such that

$$
\text { cardinality }\left(\sigma^{\prime} \cap\{x \mid x \leqq F(y)\}\right) \geqq y
$$

for infinitely many $y$, then $\sigma$ is not $h h$-simple. Thus $\sigma$ is not $h h$-simple if there exists a strongly r.e. sequence $\left\{\alpha_{i}\right\}$ of disjoint finite sets such that

$$
\text { cardinality }\left(\sigma^{\prime} \cap \alpha_{y}\right) \geqq y
$$

for infinitely many $y$. There is also an easy proof of this last result via Theorem 3 of the present paper.

A set is called r-maximal if it is r.e. and if no recursive set splits its complement nontrivially. It is easy to show that every $r$-maximal set is $h$-simple and that on the other hand there are $h$-simple sets which are neither $h h$-simple nor $r$-maximal (for example, every nonrecursive r.e. set with a retraceable complement is such a set using a theorem in [11]). Also, it follows from Theorem 3 of the present paper that an $r$-maximal set can have at most one $h h$-simple superset (modulo finite sets) and that must be maximal. It follows easily that a set is a nonmaximal, $r$-maximal set with an $h h$-simple superset if and only if it is a major subset of a maximal set. The following theorem which was proved independently by $\mathrm{R}$. W. Robinson shows that there are $r$-maximal sets which are not even subsets of maximal sets.

THEOREM 8. There is an r-maximal set which has no hh-simple superset.

Proof. Let $\chi_{i}, \chi_{i, s}$ be just as in the proof of the last theorem. Let $\left\{\alpha_{i}\right\}$ be an r.e. sequence of disjoint finite sets such that $\alpha_{i}$ has cardinality $2^{i} \cdot i$ and such that $\bigcup\left\{\alpha_{i} \mid i \geqq 0\right\}=\nu$. In step $s$ we define a strictly increasing sequence of numbers 
$p_{0, s}, p_{1, s}, \ldots$, and a strongly r.e. sequence $\beta_{0, s}, \beta_{1, s}, \ldots$ of disjoint finite sets. We define an auxiliary function $W$ by

$$
W(x, y, s)=\sum\left\{2^{y-z} \mid \beta_{x, s} \subseteq \chi_{z, s} \& z \leqq y\right\} .
$$

Let $\pi_{s}$ denote the set $\left\{p_{i, s} \mid i \geqq 0\right\}$.

We say that $n$ requires attention at step $s+1$ if $n \in \pi_{s}$ and there exists $m<n$ such that

$$
\text { 2(cardinality } \left.\beta_{n, s} \cap \chi_{m, s}\right) \geqq \text { cardinality } \beta_{n, s}
$$

and such that $\beta_{n, s} \nsubseteq \chi_{m, s}$. The construction is now as follows.

Step 0. Define $\beta_{i, 0}=\alpha_{i}$ and $p_{i, 0}=i$ for all $i$.

Step $s+1$. The step consists of two parts:

Part 1. Let $n$ be the least number if any which requires attention at step $s+1$ and let the corresponding $m$ be chosen as small as possible. We set $\beta_{n, s+1}=\beta_{n, s}$ $\cap \chi_{m, s}$ and say that $n$ receives attention through $m$ at step $s+1$. For $i \neq n$, or for all $i$ if $n$ is undefined, we set $\beta_{i, s+1}=\beta_{i, s}$.

Part 2. Define the sequence $p_{0, s+1}, p_{1, s+1}, \ldots$, by

$$
p_{0, s+1}=\min \left\{x \mid x \in \pi_{s} \& W(x, 0, s)=\max \left\{W(y, 0, s) \mid y \in \pi_{s}\right\}\right\}
$$

and for all $i>0$,

$$
\begin{aligned}
& p_{i, s+1}=\min \left\{x \mid x \in \pi_{s} \& x>p_{i-1, s+1}\right. \\
& \left.\quad \& W(x, i, s)=\max \left\{W(y, i, s) \mid y \in \pi_{s} \& y>p_{i-1, s+1}\right\}\right\} .
\end{aligned}
$$

This completes the construction. It is easy to see that it is effective in the sense that $p_{i, s}$ is a recursive function of $i, s$ and $\left\{\beta_{i, s}\right\}$ is a strongly r.e. double sequence of finite sets. We need only recall that $\bigcup\left\{\chi_{i, s} \mid i \geqq 0\right\}$ has cardinality $\leqq s+1$ and notice that $\left\{i \mid \beta_{i, s} \neq \alpha_{i}\right\}$ has cardinality $\leqq s$. Thus for fixed $s$ there are only a finite number of pairs $(x, y)$ such that $W(x, y, s) \neq 0$ and we can find which they are.

We note from Part 1 of step $s+1$ that $\beta_{i, s+1} \subseteq \beta_{i, s}$ for all $i$; denote $\lim _{x} \beta_{i, x}$ by $\beta_{i}$. It follows that for fixed $x, y$ we have $W(x, y, s)$ nondecreasing with $s$. Further, it is immediate from the definition of $W$ that $W(x, y, s)<2^{y+1}$. Hence $\lim _{z} W(x, y, z)$ exists and we denote it by $W(x, y)$.

It is easy to prove by induction on $i$ that $\lim _{x} p_{i, x}$ exists, call it $p_{i}$. It follows that $\lim _{x} \pi_{x}$ exists, call it $\pi$. Again by induction on $i, \lim _{x} W\left(p_{x}, i\right)$ exists. These inductions are similar to and somewhat easier than several occurring earlier in the paper and so we leave them to the reader.

Consider the sequence of sets $\beta_{i, 0}, \beta_{i, 1}, \ldots$ If $\beta_{i, s} \neq \beta_{i, s+1}$ then there exists $m<i$ such that $i$ receives attention through $m$ at step $s+1$. When this happens we see that $\beta_{i, s} \nsubseteq \chi_{m, s}$,

$$
\text { 2(cardinality } \left.\beta_{i, s} \cap \chi_{m, s}\right) \geqq \text { cardinality } \beta_{i, s},
$$

and $\beta_{i, s+1}=\beta_{i, s} \cap \chi_{m, s}$. Observe that the cardinality of $\beta_{i, s+1}$ is not less than half 
that of $\beta_{i, s}$, and that $\beta_{i, t} \subseteq \chi_{m, t}$ for all $t>s$. It follows that $i$ receives attention through $m$ at most one step. Hence

$$
\text { cardinality } \beta_{i} \geqq i \cdot 2^{i-j} \geqq i,
$$

where

$$
j=\text { cardinality }\{x \mid x<i \& i \text { receives attention through } x\} \text {. }
$$

We now show that $\eta=\left(\bigcup\left\{\beta_{x} \mid x \in \pi\right\}\right)^{\prime}$ has all the required properties. First of all, $\eta$ is r.e. because $\pi^{\prime}$ is r.e. and because for each $i$ the sequence $\beta_{i, 0}, \beta_{i, 1}, \ldots$ is decreasing. Let $m$ be fixed, then since $\lim _{x} W\left(p_{x}, m\right)$ exists we have $W(p, m)$ constant for all sufficiently large $p$ in $\pi$. Suppose $W(p, m)$ is odd for all sufficiently large $p$ in $\pi$, then $\beta_{p} \subseteq \chi_{m}$ for all sufficiently large $p$ in $\pi$. Thus in this case $\eta \cup \chi_{m}$ is cofinite. Suppose $W(p, m)$ is even for all sufficiently large $p$ in $\pi$, then for all sufficiently large $p$ in $\pi$ there is no step at which $p$ receives attention through $m$ and also $\beta_{p} \nsubseteq \chi_{m}$. It follows in this case that for any sufficiently large $p$ in $\pi$ and all sufficiently large $s$ (depending on the choice of $p$ ) we have $p \in \pi_{s}, m<p, \beta_{p, s} \nsubseteq \chi_{m, s}$ and yet $p$ does not require attention through $m$ at step $s+1$. Hence for $p$ sufficiently large in $\pi$ we have

$$
\text { 2(cardinality } \left.\beta_{p, s} \cap \chi_{m, s}\right)<\text { cardinality } \beta_{p, s}
$$

for all sufficiently large $s$. It follows that

$$
\text { 2(cardinality } \left.\beta_{p}-\chi_{m}\right)>\text { cardinality } \beta_{p} \text {. }
$$

But from the first half of (23) the right-hand side is easily seen to be $\geqq 2 p$. Thus for all sufficiently large $p$ in $\pi, \alpha_{p}-\left(\eta \cup \chi_{m}\right)$ has cardinality $>p$. From the result quoted just before the statement of the theorem we have $\eta \cup \chi_{m}$ not $h h$-simple.

Finally, suppose $\chi_{m}, \chi_{n}$ are disjoint recursive sets with union $v$ which split the complement of $\eta$ nontrivially. We see at once that $W(p, m)$ and $W(p, n)$ are both even for all sufficiently large $p$ in $\pi$. Otherwise, we should have $\eta \cup \chi_{m}$ or $\eta \cup \chi_{n}$ cofinite. From (24), $\beta_{p}-\chi_{m}$ and $\beta_{p}-\chi_{n}$ both have cardinality exceeding half the cardinality of $\beta_{p}$ for all sufficiently large $p$ in $\pi$. Hence $\left(\chi_{m} \cup \chi_{n}\right)^{\prime}$ is nonempty; contradiction. This completes the proof of the theorem.

One obvious component of the above construction is the maximal set construction of Friedberg. For, if we use only Part 2 of step $s+1$ and redefine $W$ by

$$
W(x, y, s)=\sum\left\{2^{y-z} \mid x \in \chi_{z, s} \& z \leqq y\right\}
$$

then $\pi^{\prime}$ is a maximal r.e. set. For any r.e. set $\eta$ let $\mathscr{L}_{r}(\eta)$ be the lattice of sets of the form $\eta \cup \rho$ where $\rho$ is recursive. It is easy to see that $\mathscr{L}_{r}^{*}(\eta)$ is a $\exists \forall \exists$-Boolean algebra. Replacing the maximal set construction by that of the last section the following generalisation of the last theorem can be obtained: let $\mathscr{A}$ be any $\exists \forall \exists-$ Boolean algebra there exists an r.e. set $\alpha$ with no hh-simple superset such that $\mathscr{L}_{r}^{*}(\alpha) \cong \mathscr{A}$. 
6. Conclusion $\left({ }^{1}\right)$. The problem which motivated this paper, that of classifying the decision problem of $\mathscr{R}$, is still far from solution. We now discuss how the programme begun in this paper may be continued. We shall also mention some recent theorems concerning other elementary theories which arise in recursive function theory.

One of the most interesting questions suggested by the present work is: what conditions must a distributive $\exists \forall \exists$-lattice $\mathscr{A}$ satisfy for there to exist an r.e. set $\alpha$ such that $\mathscr{L}^{*}(\alpha) \cong \mathscr{A}$ ? Part of the answer is clearly contained in Theorem 4 , but not the whole answer, as is illustrated by two theorems obtained by the author since this paper was written:

T1. Given r.e. sets $\alpha, \beta$ with $\alpha \subseteq \beta$ and $\alpha \cup \beta^{\prime}$ not r.e. we can effectively find r.e. sets $\beta_{1}, \beta_{2}$ such that $\beta_{1} \cup \beta_{2}=\beta, \beta_{1} \cap \beta_{2}=\alpha$, and such that $\beta_{1}-\alpha, \beta_{2}-\alpha$ are both infinite.

T2. Let $\alpha, \beta$ be r.e. sets such that $\alpha \subseteq \beta$ and $\alpha \cup \beta^{\prime}$ is not r.e. From $\alpha, \beta$ can be effectively found an r.e. set $\gamma$ such that $\gamma \supseteq \alpha$ and $\gamma$ is a major subset of $\beta$.

A stronger theorem than $\mathrm{T} 1$ whose conclusion has the additional stipulation that $\alpha \cup \beta_{1}^{\prime}, \alpha \cup \beta_{2}^{\prime}$ be non-r.e. has been obtained independently by Owings. His theorem can be easily deduced from T1 and T2. At present we have no conjecture as to the extent of the requirements that $\mathscr{A}$ must satisfy to be isomorphic to $\mathscr{L}^{*}(\alpha)$ for some r.e. $\alpha$. It seems to the author that the next step is to attempt to construct an r.e. set $\alpha$ with no $h h$-simple superset such that $\mathscr{L}^{*}(\alpha)$ is known independently. However, we expect that to achieve this the technique of Friedberg's maximal set construction will have to be replaced by one with a more complex structure.

The author's own more recent work has been directed towards building up piecemeal a decision procedure for $\mathscr{R}^{*}$. For this purpose it is convenient to replace the sole relative symbol $Q($, ) used here by the following set: function symbols for union, intersection, and complement together with two unary relative symbols $R, E$. In the interpretation the quantifiers range over $\mathscr{R}^{*} ; R(z), E(z)$ mean $z \in \mathscr{R}^{*}$, $z=\{\varnothing\}$ respectively. Using the methods of this paper we have obtained a decision procedure for the $\forall \exists$-sentences of this new language in $\mathscr{R}^{*}$. The three quantifier class can be approached in the same way and a systematic treatment of it would give us a much deeper insight into the structure of $\mathscr{R}^{*}$ than we have at present.

A problem left over from $\S 4$ is that of determining for which sets $\mu$ the lattice $\mathscr{H}^{\mu}$ of sets $h h$-simple relative to $\mu$ is isomorphic to $\mathscr{H}^{\phi}$. In particular, is $\mathscr{H}^{\mu}$ isomorphic to $\mathscr{H}^{\phi}$ for every arithmetic set $\mu$ ?

Another and quite different line which might be fruitful is the investigation of the lattice of r.e. sets modulo simple sets. We write $\alpha \approx \beta$ just if $(\alpha-\beta) \cup(\beta-\alpha)$ is immune. It is easy to check that $\approx$ is a congruence relation on $\mathscr{R}$. The lattice formed

( ${ }^{1}$ In making corrections to the paper this section was entirely rewritten to bring it up to date, November 10, 1966. 
by the congruence classes into which $\approx$ divides $\mathscr{R}$ is called the lattice of r.e. sets modulo simple sets. Can any interesting structure results be obtained for this lattice?

One specific question about maximal sets is: are there any elementary classes of maximal sets other than the empty class and the class of all maximal sets?

It is natural to ask the same kind of questions about the elementary theories of other structures which are studied in recursive function theory. The cases that we know something about are the following three:

(i) The elementary theory of many-one degrees has been shown nonaxiomatisable by the author. This is proved by showing that any countable distributive lattice with greatest and least elements is isomorphic to an initial segment of the many-one degrees. The method can be refined to show that the elementary theory of r.e. many-one degrees is also nonaxiomatisable.

(ii) For Turing degrees D. F. Hugill has shown that there is an initial segment isomorphic to any countable linearly ordered set with least member. His method extends Spector's construction of a minimal degree, and will probably also work in the case where "distributive lattice" replaces "linearly ordered set". If this turns out to be the case, it will follow as for the many-one degrees that the elementary theory is nonaxiomatisable.

(iii) Let $\left\{\psi_{i}\right\},\left\{\chi_{i}\right\}$ be two standard enumerations of the class of r.e. sets, and let $F, G$ be the corresponding binary relations, i.e., $F(x, y) \Leftrightarrow x \in \psi_{y}$ and $G(x, y)$ $\Leftrightarrow x \in \chi_{y}$. It can be shown that there is a recursive permutation $\pi$ such that $F(x, y)$ is equivalent to $G(\pi(x), \pi(y))$. We conclude that the elementary theory of $F$ is independent of which standard enumeration we choose. This elementary theory which we denote by $S E$ can also be shown to be nonaxiomatisable.

Added April 10, 1967. I am grateful to C. Jockusch and A. Manaster for noticing an error in my proof that $\mathscr{H}^{*}$ the lattice of cofinite and $h h$-simple sets modulo finite sets is decidable. That proof has been deleted from the paper. However, Ershov [2] has described all complete extensions of the elementary theory $R D$ of relatively complemented distributive lattices and shown them all to be decidable. Thus the decidability of $\mathscr{H}^{*}$ is a corollary of Theorem 3. From Theorem 6 one can deduce exactly which complete extension of $R D$ is the elementary theory of $\mathscr{H}^{*}$; it turns out to be the one which is richest in structure.

\section{REFERENCES}

1. M. Davis, Computability and unsolvability, McGraw-Hill, New York, 1958.

2. Ju. L. Ershov, Decidability of the elementary theory of relatively complemented distributive lattices and of the theory of filters, Algebra i Logika Sem. 3 (1964), 17-38. (Russian)

3. R. M. Friedberg, Three theorems on recursive enumeration, J. Symbolic Logic 23 (1958), 309-316.

4. - - 4-quantifier completeness: a Banach-Mazur functional not uniformly partial recursive, Bull. Acad. Polon. Sci. Sér. Sci. Math. Astronom. Phys. 6 (1958), 1-5.

5. D. A. Martin, A theorem on hyperhypersimple sets, J. Symbolic Logic 28 (1963), 273-277. 
6. - Classes of recursively enumerable sets and degrees of unsolvability, Z. Math. Logik Grundlagen Math. 12 (1966), 295-310.

7. J. Myhill, Review of [10], Math. Reviews 26 (1963), 691, \#3598.

8. E. L. Post, Recursively enumerable sets of positive integers and their decision problems, Bull. Amer. Math. Soc. 50 (1944), 284-316.

9. A. Robinson, Introduction to model theory and the metamathematics of algebra, NorthHolland, Amsterdam, 1963.

10. C. E. M. Yates, Recursively enumerable sets and retracing functions, Z. Math. Logik Grundlagen Math. 8 (1962), 331-345.

11. - On the degrees of index sets, Trans. Amer. Math. Soc. 121 (1966), 309-328.

SIMON FRASER UNIVERSITY,

Burnaby 2, British Columbia 